\title{
CENTRAL ASPECTS OF SKEW TRANSLATION QUADRANGLES, 1
}

\author{
KOEN THAS \\ Dedicated to my dear friend Ernie shult
}

abstract. Modulo a combination of duality, translation duality or Payne integration, every known finite generalized quadrangle except for the Hermitian quadrangles $\mathcal{H}\left(4, q^{2}\right)$, is an elation generalized quadrangle for which the elation point is a center of symmetry - that is, is a "skew translation generalized quadrangle" (STGQ). In this series of papers, we classify and characterize skew translation generalized quadrangles. In the first installment of the series,

(1) we obtain the rather surprising result that any skew translation quadrangle of finite odd order $(s, s)$ is a symplectic quadrangle;

(2) we determine all finite skew translation quadrangles with distinct elation groups (a problem posed by Payne in a less general setting);

(3) we develop a structure theory for root-elations of skew translation quadrangles which will also be used in further parts, and which essentially tells us that a very general class of skew translation quadrangles admits the theoretical maximal number of root-elations for each member, and hence all members are "central" (the main property needed to control STGQs, as which will be shown throughout);

(4) we show that finite "generic STGQs," a class of STGQs which generalizes the class of the previous item (but does not contain it by definition), have the expected paramters. We conjecture that the classes of (3) and (4) contain all STGQs.

\section{CONTENTS}

1. Introduction 2

2. Some preliminaries 6

\begin{tabular}{llr}
\hline 3. & STGQs & 10
\end{tabular}

\begin{tabular}{ll}
\hline Part I - Structure theory & 14
\end{tabular}

\begin{tabular}{lll}
\hline 4. & Local Moufang conditions & 14 \\
\hline
\end{tabular}

\begin{tabular}{lll}
\hline 5. & Fix point theory and structure & 17
\end{tabular}

\begin{tabular}{lll}
\hline 6. & EGQs of order $\left(t^{2}, t\right)$ with $(*)$ are STGQs & 21
\end{tabular}

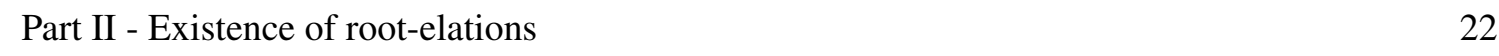

$\begin{array}{lll}7 . & \text { Root-elations } & 22\end{array}$

$\begin{array}{ll}\text { Part III - Square odd STGQs - classification } & 27\end{array}$

\begin{tabular}{lll}
\hline 8. & Square STGQs of odd order - classification & 27
\end{tabular}

\begin{tabular}{lll}
\hline 9. Combinatorial lemma & 30
\end{tabular}

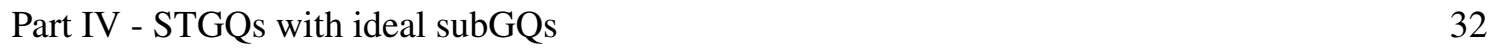

10. Tame groups and twisting 32

11. STGQs with more than one elation group are classical 33

2000 Mathematics Subject Classification. 05B25, 05E20, 20B10, 20B25, 20D15, 51E12, 51E14, 51E20. 
Part V - Generic STGQs 38

\begin{tabular}{lll}
\hline 12. & Generic STGQS & 38
\end{tabular}

References

\section{INTRODUCTION}

Let $L$ be a nilpotent $p$-group of class 2 , and suppose that $\Phi(L)=[L, L] \leq Z(L)$, where $Z(L)$ is the center of $L$, and $\Phi(L)$ is the Frattini subgroup of $L$. The latter is by definition the intersection of all maximal subgroups of $L$, and since $L$ is a $p$-group, it holds that $\Phi(L)=L^{p}[L, L]$. Define a map $\chi$ as follows:

$$
\chi: V \times V \longmapsto[L, L]:(a \Phi(L), b \Phi(L)) \longmapsto[a, b],
$$

where $V:=L / \Phi(L)$ is seen as an $\mathbb{F}_{p}$-vector space. Then $\chi$ is a bi-additive map. A fundamental question is to find minimal conditions on $L$ to conclude that $\chi$ is a bilinear form over $\mathbb{F}_{q}$, where $|\Phi(L)|=q$, a power of $p$. (So $\chi$ then indicates how scalar multiplication works.) For instance, in [32], Stroth formulated conditions on 2-groups of class 2 (which were later corrected in [23]) to force $\chi$ to be a form. Once one knows that $\chi$ is a bilinear form, one can use the geometry of $(V \times V, \chi)$ to study the structure of $L$.

For incidence geometry, such questions are very important. One of the most studied classes of buildings is the class of so-called "flock quadrangles," and such quadrangles carry a subgroup $K$ of the automorphism group, for which (in the finite case) $|K|=q^{5}$ for some prime power $q$, $\Phi(K)=[K, K]=Z(K)$ is elementary abelian of size $q$, and the map $\chi$ indeed is a (non-singular, alternating) bilinear form ("BAN-form"). We have that $K \cong \mathcal{H}_{2}(q)$ - the 5 -dimensional Heisenberg group over $\mathbb{F}_{q}$. The group $K$ comes with two families $\mathcal{F}$ and $\mathcal{F}^{*}$ of subgroups which carry the geometric information of the quadrangle (i.e., the quadrangle can be described in terms of cosets of elements of $\left.\mathcal{F} \cup \mathcal{F}^{*}\right)$. The elements of $\mathcal{F} \cup \mathcal{F}^{*}$ satisfy certain properties, and vice versa, groups $K$ with families $\mathcal{F}$ and $\mathcal{F}^{*}$ of subgroups satisfying these properties act as certain automorphism groups of generalized quadrangles defined through this coset geometry construction. The pair $\left(\mathcal{F}, \mathcal{F}^{*}\right)$ is called a Kantor family in $K$. In [48], I proved the following converse.

Theorem 1.1 ([48]). Suppose $H$ is a p-group of order $q^{5}$ for which $Z(H)=\Phi(H)=[H, H]$ is elementary abelian of order $q$. Suppose $H$ admits a Kantor family of type $\left(q^{2}, q\right)$, and suppose $\chi$ defines a BAN-form over $\mathbb{F}_{q}$. Then $H \cong \mathcal{H}_{2}(q)$, and the corresponding generalized quadrangle $\mathcal{S}$ of order $\left(q^{2}, q\right)$ is a flock quadrangle.

The main idea behind the proof is that the BAN-form produces a symplectic polar space (which is another building) in the projective space coming from $V$, and these geometries are well understood. Then going back to the quadrangle, one proves a characterizing property for flock quadrangles.

For a flock quadrangle (of order $\left(q^{2}, q\right)$ ), any defining Kantor family $\left(\mathcal{F}, \mathcal{F}^{*}\right)$ in $\mathcal{H}_{2}(q)$ has the property that for $A^{*}, B^{*} \neq A^{*} \in \mathcal{F}^{*}, A^{*} \cap B^{*}=: \mathbb{S}$ is a normal subgroup of $\mathcal{H}_{2}(q)$ which is independent of the choice of $A^{*}, B^{*}$. Generalized quadrangles arising from Kantor families with this additional property are, by definition, skew elation generalized quadrangles ("STGQs"). This abstract class of quadrangles is very general: except for the quadrangles associated to Hermitian varieties $\mathcal{H}\left(4, q^{2}\right)$, every known finite generalized quadrangle is an STGQ up to a combination 
of duality, translation duality or so-called "Payne integration.'11 So understanding the structure of STGQs (possibly up to the point of classification) is one of the main foundational challenges in the theory of rank two buildings. And this is precisely the aim of the present series of papers.

1.1. From BN-Pairs to local Moufang conditions. A famous result of Tits [53] states that an axiomatic spherical building of rank at least 3 can be constructed through a group coset geometry construction from a so-called "(group $G$ with a) BN-pair" (for the special case of axiomatic projective spaces, this boils down to the classical Veblen-Young theorem which says that if the dimension is at least 3 , the space can be coordinatized by a vector space over a skew field, and $G$ contains the little projective group of that vector space). In the other direction, each group with a BN-pair gives rise to a building. We mention that a group $G$ is said to have a $B N$-pair $(B, N)$, where $B, N$ are subgroups of $G$, if the following properties are satisfied:

(BN1) $\langle B, N\rangle=G$;

(BN2) $H=B \cap N \triangleleft N$ and $N / H=W$ is a Coxeter group with distinct generators $s_{1}, s_{2}, \ldots, s_{n}$;

(BN3) $B s_{i} B w B \subseteq B w B \cup B s_{i} w B$ whenever $w \in W$ and $i \in\{1,2, \ldots, n\}$;

(BN4) $s_{i} B s_{i} \neq B$ for all $i \in\{1,2, \ldots, n\}$.

The subgroup $B$, respectively the quotient group $W$, is a Borel subgroup, respectively the Weyl group, of $(B, N)$ (where we note that a group can have more than one $(B, N)$-pair). The natural number $n$ is called the rank of the BN-pair; when the rank is 2 the Weyl group $N /(B \cap N)$ is a dihedral group of size $2 m$ for some $m$. We say that the BN-pair $(B, N)$ is of type $B_{2}$ if $W$ is a dihedral group of size 8 .

From the data given in (BN1)-(BN4), one constructs maximal parabolics $P_{i}$, one for each generator $s_{i}$, and the element set of the associated building $\mathcal{B}(G ; B, N)$ consists of left cosets of the maximal parabolics, while such elements are incident if they have nontrivial intersection. Then $G$ acts naturally as an automorphism group on $\mathcal{B}(G ; B, N)$ by left multiplication. Sphericality expresses the fact that $W$ is a finite group (which translates geometrically into the fact that apartments are finite). One can prove that higher rank buildings can be described completely by their rank 2 residues [53], which are buildings of rank 2, i.e., generalized polygons [55].

In [53], Tits obtains a splitness result for each BN-pair associated to a spherical building of rank at least three; interpreted on the rank 2 residues, one obtains that the residual polygons are Moufang polygons. Much later, the classification of Moufang polygons by Tits and Weiss [54] became a flagship in incidence geometry, and showed to be more difficult than the classification of higher rank spherical BN-pairs (having Tits's classification [53] as a consequence). The Moufang condition and local variations has become the central group theoretical condition in incidence geometry, as (e.g.) the well-known and much-studied notions of translation plane [19] (planes that admit all local Moufang conditions at a line), elation quadrangle [47] (the natural generalization to quadrangles, which enabled Payne and Thas to give a new proof of the classification of finite Moufang quadrangles [26, 27]), translation quadrangle [37] (another "abelian" variation), Moufang polygon [54], etc. show. The reader observes that in the rank 2 case, one needs to impose extra (for instance group theoretical) conditions in order to have a grasp on these objects, in marked contrast with the higher rank case where large automorphism groups are always present, as we have seen. The concept of free construction is just one of the many examples which underlines this view.

Although in the theory of finite generalized polygons, local Moufang conditions have not produced new examples if the gonality is at least 6 , the two other cases (gonality 3 and 4 ) have proved to be conceptually different: in fact, recent work has shown that especially in gonality 4 (i.e., for generalized quadrangles) automorphism groups can behave in a deep and unpredictable way.

\footnotetext{
${ }^{1}$ This is a term coined by the author of the present paper. Given a generalized quadrangle $\Gamma$ of finite order $s$ and with regular point $x$, the Payne derived quadrangle with respect to $x$ is a quadrangle $\mathcal{P}(\Gamma, x)$ of order $(s-1, s+1)$. I define a "Payne integral" of $\mathcal{P}(\Gamma, x)$ as any quadrangle $\Gamma^{\prime}$ of order $s$ and with regular point $x^{\prime}$ for which $\mathcal{P}\left(\Gamma^{\prime}, x^{\prime}\right) \cong \mathcal{P}(\Gamma, x)$. Two such integrals do not have to be isomorphic. And $\int \mathcal{P}(\Gamma, x) d x$ denotes the set of all such integrals.
} 
Even more, in the last twenty years, classes of examples have been (re-)discovered which satisfy a combination of local Moufang conditions, though not satisfying the global Moufang condition. Perhaps the main role has been played (in one way or another) by Payne's MSTGQs [24] (see $\S \S$ 4.1), which is a very rich class containing the important flock quadrangles, and each of its members is "locally very Moufang." All these examples are examples of STGQs, and by recent work of the author and also by work presented here, one observes that the class of STGQs strictly contains the MSTGQs.

In fact, this entire paper and its sequels should be seen as a new vista on local Moufang conditions in finite and infinite generalized quadrangle theory. More precisely, we call an STGQ $\mathcal{S}^{x}$ central if each symmetry about $x$ is contained in the center of any elation group with respect to $x$. We refer to "Centrality conjecture" as the conjecture that any STGQ is central. The Centrality conjecture is one the main actors in this series of papers - it will be solved for (*)-STGQs (cf. $\$ \S 5.1$ for a detailed discussion on this property) in the present paper (the notable exception being the case $s=t$ even). Centrality gives rise to a number of local Moufang conditions (and vice versa), which enable one to control this large class of quadrangles.

1.2. The known results. The first positive result towards STGQ classification is the one, independently obtained by Chen and Hachenberger, on the parameters of an STGQ (solving a well-known conjecture of Payne):

Theorem 1.2 (Chen [9], see also [47]; Hachenberger [16]). The parameters $s, t$ of an $S T G Q$ are powers of the same prime.

A more recent one, by Bamberg, Penttila and Schneider, is, by my knowledge, a first "complete" classification result, after assuming conditions on the parameters:

Theorem 1.3 (Bamberg, Penttila and Schneider [3]). An STGQ of order $(s, p)$, with p a prime, is either classical or a flock quadrangle.

In fact, in [3] the same conclusion is proved for EGQs, but it follows easily from the assumptions that the quadrangle is an STGQ. The fact that $t=p$ is a prime forces that either $s=t$ or $s=t^{2}$. The first case is known to lead to a quadric; in the second case the authors show that the aforementioned bi-additive map is a BAN-form.

The final classification result we know of (besides Theorem[1.1), is the very elegant

Theorem 1.4 (Ghinelli [14]). A square STGQ with odd order and of symplectic type is classical.

One of the results of the present paper tells us that square STGQs with odd order always are of symplectic type, thus finishing the classification of odd square order STGQs (we indicate an independent argument why the STGQ then is classical). I announced this result back in May 2007 (see the note [50] for some chronological details). In fact, all (*)-STGQs (except for even square ones) will be shown to be of symplectic type in a geometric sense.

Remark 1.5 (Quadrangular representations). We say that a group $K$ has a (faithful) quadrangular representation (of type $(s, t)$ ) if there is a generalized quadrangle $\mathcal{S}$, a point $x$ of $\mathcal{S}$, and an injection $\iota: K \longmapsto \operatorname{Aut}(\mathcal{S})$ such that $\left(\mathcal{S}^{x}, \iota(K)\right)$ is an EGQ (of order $(s, t)$ ). The representation is skew if $\left(\mathcal{S}^{x}, \iota(K)\right)$ is an STGQ. One of the goals in this series is to classify and understand (finite and infinite) groups which have a skew quadrangular representation, as well as the isomorphism classes of possible representations of such groups. Another fundamental question that arises is which groups (if any) can have quadrangular representations of different type. The only examples I know of are certain elementary abelian groups. (Similar questions arise in the theory of Singer groups for projective spaces. In that context, existence of groups which act as Singer groups on 
finite projective spaces of different dimensions yield strange number theoretical identities.)

1.3. Structure of the present paper. The paper is organized as follows.

0 - In a first part (0) of the paper we introduce some elementary combinatorial features which will be used frequently throughout. By no means we are complete - we only define the language which is most often used, and refer to other sources on the way, if needed. The concept of skew translation generalized quadrangle is described, as are the known (classes of) examples of skew translation generalized quadrangles in some detail (we also mention the known elation groups which arise). These descriptions partly come from very recent results, and contain new results on the local structure of one of these elation groups which sheds new light on the possible actions of skew elation groups.

I- In a second part (I), we develop a basic structure theory for fixed points configurations for elements of skew elation groups. We introduce a crucial property (called "(*)"), which is enjoyed by most of the known STGQs. However, the aforementioned local result shows that (surprisingly) there are examples which do not have (*). This obstruction motivates us to introduce "generic STGQs" later in the paper. As a corollary of the fixed point theory, we show that once an STGQ has $(*)$, we can deduce precise (and very strong, as the reader will see) information on the number of fixed lines of elation group elements. This is one of the main tools for the next part.

II- In that part (II), we discuss the existence of root-elations in STGQs, mostly in the case that (*) holds. The aforementioned fixed line theorem allows us to show that (*)-STGQs (assumed that when the STGQ is square, we are not in even characteristic) satisfy all local Moufang conditions with respect to the elation point. We then deduce that such STGQs are central. We indicate several entirely different ways to do this, each with its own advantages.

III- Next, we obtain one of the central results of this paper (and of the entire series): we show that all STGQs $\left(\mathcal{S}^{x}, K\right)$ of odd square order are isomorphic to $\left(\mathcal{W}(t), \mathcal{H}_{1}(t)\right)$, that is, all come from a symplectic polar space in 3 dimensions. So the only groups with a faithful skew quadrangular representation of type $(t, t), t$ odd, are 3 -dimensional Heisenberg groups. This generalizes Theorem 1.4 to a large extent.

IV - In a fifth part, we consider STGQs which have ideal subGQs (through the elation point), and show that this assumption is enough to deduce strong structural information, including local Moufang conditions and centrality. We apply this theory to consider STGQs which admit different elation groups (after a question of Payne), and solve his question by showing that such STGQs arise from Hermitian varieties. Also, further in the paper and in the series, the subGQ theory will be highly useful.

V-Finally, we study the category $\mathcal{G}$ of "generic STGQs" - STGQs which do not enjoy (*), but which we enhance with two very general natural properties that generalize $\left(^{*}\right)$. (In fact, one of the main aims is to show that the category of generic STGQs coincides with that of non-(*) STGQs.) We show that every object of $\mathcal{G}$ has ideal subGQs, so that the previous part can be applied. As a corollary, we show that for a (*)-STGQ or generic STGQ, either the possible parameters have the form $(t, t)$ or $\left(t^{2}, t\right)$ (with $t$ a prime power), or the STGQ is "abelian" (in a very specific case). This is a first big step which aims, eventually, at showing that the same arithmetic conclusion holds for any STGQ (Main Parameter Conjecture). 
1.4. Acknowledgements. I presented some of the main results of this paper as a series of (then partly conjectural) results in a lecture, at the conference "Buildings and Groups" (Ghent, May 2007), which was organized by Peter Abramenko, Bernhard Mühlherr and Hendrik Van Maldeghem. Some of the ideas presented at that conference were developed when I was hosted, together with S. De Winter and E. E. Shult, by the Mathematisches Forschungsinstitut Oberwolfach (MFO) in the Research in Pairs program (April 2007). A short synopsis of the chronology can be found in the note [50].

I want to thank an anonymous referee for a number of helpful comments and suggestions on a draft of this paper. Finally, I wish to thank Stanley E. Payne for sending me numerous remarks and questions, which helped me to put the paper into its final shape.

\section{SOME PRELIMINARIES}

We start this section with introducing some combinatorial and group theoretical notions.

2.1. Elementary combinatorial preliminaries. We tersely review some basic notions taken from the theory of generalized quadrangles, for the sake of convenience.

2.1.1. Finite generalized quadrangles. Let $\Gamma$ be a thick generalized quadrangle (GQ). It is a rank 2 geometry $\Gamma=(\mathcal{P}, \mathcal{B}, I)$ (where we call the elements of $\mathcal{P}$ "points" and those of $\mathcal{B}$ "lines") such that the following axioms are satisfied:

(a) there are no ordinary digons and triangles contained in $\Gamma$;

(b) each two elements of $\mathcal{P} \cup \mathcal{B}$ are contained in an ordinary quadrangle;

(c) there exists an ordinary pentagon.

It can be shown that there are exist constants $s$ and $t$ such that each point is incident with $t+1$ lines and each line is incident with $s+1$ points. We say that $(s, t)$ is the order of $\Gamma$. If we say, in general, that a rank 2 geometry has order $(s, t)$, it means the same thing.

A rank 2 geometry satisfying (a) and (b) but not (c) is a thin GQ. If (c) is satisfied, then the GQ is thick, since $s$ and $t$ have the property that they are both at least 2 . An ordinary quadrangle is just a thin GQ of order $(1,1)$; we call an ordinary quadrangle in $\Gamma$ also "apartment" (of $\Gamma$ ).

If $s=t$, then $\mathcal{S}$ is also said to be of order $s$; we call GQs of order $s$ for some $s$ square generalized quadrangles.

Suppose $p \pm L$. Then by $\operatorname{proj}_{L} p$, we denote the unique point on $L$ collinear with $p$. Dually, $\operatorname{proj}_{p} L$ is the unique line incident with $p$ concurrent with $L$.

2.1.2. Roots and i-roots. Let $A$ be an apartment of a GQ $\Gamma$. A root $\gamma$ of $A$ is a set of 5 different elements $e_{0}, \ldots, e_{4}$ in $A$ such that $e_{i} \mathrm{I} e_{i+1}$ (where the indices are taken in $\{0,1,2,3,4\}$ ), and $e_{0}, e_{4}$ are the extremal elements of $\gamma$. There are two types of roots, depending on whether the extremal elements are lines or points; in the second case we speak of dual roots to make a distinction between the types. Also, a (dual) root $\gamma$ without its extremal elements - the interior of $\gamma$ - is denoted by $\dot{\gamma}$ and called (dual) i-root.

2.1.3. Point-line duality. There is a point-line duality for GQs of order $(s, t)$ for which in any definition or theorem the words "point" and "line" are interchanged and also the parameters. (If $\mathcal{S}=(\mathcal{P}, \mathcal{B}, \mathrm{I})$ is a GQ of $\operatorname{order}(s, t), \mathcal{S}^{D}=(\mathcal{B}, \mathcal{P}, \mathrm{I})$ is a GQ of order $(t, s)$.) 
2.1.4. Collinearity, concurrency and regularity. Let $u$ and $v$ be not necessarily distinct points of the GQ $\Gamma$; we write $u \sim v$ and call these points collinear, if there is some line $L$ such that $u \mathrm{I} L \mathrm{I} v$. Dually, for $U, V \in \mathcal{B}$, we write $U \sim V$ when $U$ and $V$ are concurrent. A relation $u \sim U$ or $U \sim u$ never holds if $u$ is a point and $U$ is a line. For $W \in \mathcal{P} \cup \mathcal{B}$, put

$$
W^{\perp}=\{X \in \mathcal{P} \cup \mathcal{B} \mid X \sim W\}, \text { and } W^{\perp \perp}:=\left(W^{\perp}\right)^{\perp} .
$$

For $\mathcal{A} \subseteq \mathcal{P} \cup \mathcal{B}$, let $\mathcal{A}^{\perp}:=\cap_{a \in \mathcal{A}} a^{\perp}$. Important special cases:

(*) Let $\mathcal{A}$ be a set of distinct points $\{u, v\}$; then $\left|\{u, v\}^{\perp}\right|=s+1$ or $t+1$, according as $u \sim v$ or $u \neq v$, respectively.

(*) When $u \neq v$, we have that $\left|\{u, v\}^{\perp \perp}\right|=s+1$ or $\left|\{u, v\}^{\perp \perp}\right| \leq t+1$ according as $u \sim v$ or $u \not v v$, respectively. If $u \sim v, u \neq v$, or if $u \not v$ and $\left|\{u, v\}^{\perp \perp}\right|=t+1$, we say that the pair $\{u, v\}$ is regular.

The point $u$ is regular provided $\{u, v\}$ is regular for every point $v \in \mathcal{P} \backslash\{u\}$. Regularity for lines is defined dually. One easily proves that either $s=1$ or $t \leq s$ if $\mathcal{S}$ has a regular pair of noncollinear points; see 1.3.6 of [26].

2.1.5. Antiregularity. The pair of points $\{x, y\}, x \not y$, is antiregular if $\left|\{x, y\}^{\perp} \cap z^{\perp}\right| \leq 2$ for all $z \in \mathcal{P} \backslash\{x, y\}$. The point $x$ is antiregular if $\{x, y\}$ is antiregular for each $y \in \mathcal{P} \backslash x^{\perp}$.

\subsection{Automorphism groups.}

2.2.1. Automorphisms and whorls. An automorphism of a GQ $\mathcal{S}=(\mathcal{P}, \mathcal{B}, \mathrm{I})$ is a permutation of $\mathcal{P} \cup \mathcal{B}$ which preserves $\mathcal{P}, \mathcal{B}$ and $I$. The set of automorphisms of a GQ $\mathcal{S}$ is a group, called the automorphism group of $\mathcal{S}$, which is denoted by $\operatorname{Aut}(\mathcal{S})$.

A whorl about a point $x$ is an automorphism fixing it linewise. A point $x$ is a center of transitivity provided that the group of whorls about $x$ is transitive on $\mathcal{P} \backslash p^{\perp}$.

A symmetry with center $x$ is a whorl about $x$ which fixes $x^{\perp}$ pointwise. If the GQ $\mathcal{S}$ is finite of order $(s, t)$, it is easy to see that the number of symmetries with center $x$ cannot exceed $t$, and if that number is $t, x$ must be regular point. In this case, we speak of a center of symmetry. Dually, one introduces symmetries with an axis, and axes of symmetry.

2.2.2. Moufang properties. If $\mathcal{M}$ is a subgeometry of $\Gamma$, by $\operatorname{Aut}(\Gamma)^{[\mathcal{N}]}$ we denote the subgroup of the automorphism group $\operatorname{Aut}(\Gamma)$ of $\Gamma$ which fixes every line incident with a point of $\mathcal{M}$ and every point incident with a line of $\mathcal{M}$. Now a (dual) root $\gamma$ is Moufang if $\operatorname{Aut}(\Gamma)^{[\dot{\gamma}]}$ acts transitively on the apartments containing $\gamma$. In fact, $\operatorname{Aut}(\Gamma)^{[\dot{\gamma}]}=: A(\dot{\gamma})$ then acts sharply transitively on these apartments. Once a (dual) root $\gamma$ is Moufang, all (dual) roots with interior $\dot{\gamma}$ are also Moufang, with respect to the same group $A(\dot{\gamma})$. (The latter group is uniquely defined by $\dot{\gamma}$ and the Moufang property.) In a natural way, we also use the terms "Moufang i-root" and "dual Moufang i-root," and the elements of $A(\dot{\gamma})$ are called root-elations throughout.

Now $\Gamma$ is half Moufang if all roots or all dual roots are Moufang. It is Moufang if all roots and dual roots are.

\subsection{Subquadrangles.}

2.3.1. Subquadrangles. A subquadrangle, or also $\operatorname{subGQ}, \mathcal{S}^{\prime}=\left(\mathcal{P}^{\prime}, \mathcal{B}^{\prime}, \mathrm{I}^{\prime}\right)$ of a GQ $\mathcal{S}=(\mathcal{P}, \mathcal{B}, \mathrm{I})$ is a GQ for which $\mathcal{P}^{\prime} \subseteq \mathcal{P}, \mathcal{B}^{\prime} \subseteq \mathcal{B}$, and where $I^{\prime}$ is the restriction of $I$ to $\left(\mathcal{P}^{\prime} \times \mathcal{B}^{\prime}\right) \cup\left(\mathcal{B}^{\prime} \times \mathcal{P}^{\prime}\right)$. The subGQ $\mathcal{S}^{\prime}$ is ideal if for any point $x \in \mathcal{P}^{\prime}$ we have that $\left\{L \mathrm{I} x \mid L \in \mathcal{B}^{\prime}\right\}=\{L \mathrm{I} x \mid L \in \mathcal{B}\}$. Dually, one speaks of full subGQs.

The following results will sometimes be used without further reference. 
Theorem 2.1 ([27], 2.2.1). Let $\mathcal{S}^{\prime}$ be a proper subquadrangle of order $\left(s^{\prime}, t^{\prime}\right)$ of the $G Q \mathcal{S}$ of order $(s, t)$. Then either $\mathcal{S}^{\prime}$ is full or $s \geq s^{\prime} t^{\prime}$. If $\mathcal{S}^{\prime}$ is full, then each external point of $\mathcal{S}^{\prime}$ is collinear with the $s t^{\prime}+1$ points of an ovoid of $\mathcal{S}^{\prime}$; if $s=s^{\prime} t^{\prime}$, then each external point of $\mathcal{S}^{\prime}$ is collinear with exactly $1+s^{\prime}$ points of $\mathcal{S}^{\prime}$.

Theorem 2.2 ([27], 2.2.2). Let $\mathcal{S}^{\prime}$ be a proper full subquadrangle of the $G Q \mathcal{S}$, where $\mathcal{S}$ has order $(s, t)$ and $\mathcal{S}^{\prime}$ has order $\left(s, t^{\prime}\right)\left(\right.$ so $\left.t>t^{\prime}\right)$. Then we have

(1) $t \geq s$; if $s=t$, then $t^{\prime}=1$.

(2) If $s>1$, then $t^{\prime} \leq s$; if $t^{\prime}=s \geq 2$, then $t=s^{2}$.

(3) If $s=1$, then $1 \leq t^{\prime}<t$ is the only restriction on $t^{\prime}$.

(4) If $s>1$ and $t^{\prime}>1$, then $\sqrt{s} \leq t^{\prime} \leq s$ and $s^{3 / 2} \leq t \leq s^{2}$.

(5) If $t=s^{3 / 2}>1$ and $t^{\prime}>1$, then $t^{\prime}=\sqrt{s}$.

(6) Let $\mathcal{S}^{\prime}$ have a proper full subquadrangle $\mathcal{S}^{\prime \prime}$ of order $\left(s, t^{\prime \prime}\right), s>1$. Then $t^{\prime \prime}=1, t^{\prime}=s$ and $t=s^{2}$.

A subgeometry of a generalized quadrangle $\mathcal{S}=(\mathcal{P}, \mathcal{B}, I)$ is a rank 2 geometry $\mathcal{S}^{\prime}=\left(\mathcal{P}^{\prime}, \mathcal{B}^{\prime}, \mathrm{I}^{\prime}\right)$ for which $\mathcal{P}^{\prime} \subseteq \mathcal{P}, \mathcal{B}^{\prime} \subseteq \mathcal{B}$ and $\mathrm{I} \subseteq \mathrm{I}^{\prime}$.

Theorem 2.3 ([27], 2.3.1). Let $\mathcal{S}^{\prime}=\left(\mathcal{P}^{\prime}, \mathcal{B}^{\prime}, \mathrm{I}^{\prime}\right)$ be a subgeometry of the $G Q \mathcal{S}$ of order $(s, t)$ so that the following two conditions are satisfied:

(i) if $x, y \in \mathcal{P}^{\prime}$ are distinct points of $\mathcal{S}^{\prime}$ and $L$ is a line of $\mathcal{S}$ such that $x \mathrm{I} L \mathrm{I} y$, then $L \in \mathcal{B}^{\prime}$;

(ii) each element of $\mathcal{B}^{\prime}$ is incident with $s+1$ elements of $\mathcal{P}^{\prime}$.

Then there are four possibilities:

(1) $\mathcal{S}^{\prime}$ is a dual grid, so $s=1$;

(2) the elements of $\mathcal{B}^{\prime}$ are lines which are incident with a distinguished point of $\mathcal{P}$, and $\mathcal{P}^{\prime}$ consists of those points of $\mathcal{P}$ which are incident with these lines;

(3) $\mathcal{B}^{\prime}=\emptyset$ and $\mathcal{P}^{\prime}$ is a set of pairwise noncollinear points of $\mathcal{P}$;

(4) $\mathcal{S}^{\prime}$ is a full subquadrangle of order $\left(s, t^{\prime}\right)$.

The following result is now easy to prove.

Theorem 2.4 ([27], 2.4.1). Let $\theta$ be an automorphism of the $G Q \mathcal{S}=(\mathcal{P}, \mathcal{B}, I)$ of order $(s, t)$. The subgeometry $\mathcal{S}_{\theta}=\left(\mathcal{P}_{\theta}, \mathcal{B}_{\theta}, \mathrm{I}_{\theta}\right)$ of $\mathcal{S}$ whose elements are the fixed elements of $\theta$, must be given by (at least) one of the following:

(i) $\mathcal{B}_{\theta}=\emptyset$ and $\mathcal{P}_{\theta}$ is a set of pairwise noncollinear points;

(i) $\mathcal{P}_{\theta}=\emptyset$ and $\mathcal{B}_{\theta}$ is a set of pairwise nonconcurrent lines;

(ii) $\mathcal{P}_{\theta}$ contains a point $x$ so that $y \sim x$ for each $y \in \mathcal{P}_{\theta}$, and each line of $\mathcal{B}_{\theta}$ is incident with $x$;

(ii) $^{\prime} \mathcal{B}_{\theta}$ contains a line $L$ so that $M \sim L$ for each $M \in \mathcal{B}_{\theta}$, and each point of $\mathcal{P}_{\theta}$ is incident with $L$;

(iii) $\mathcal{S}_{\theta}$ is a grid;

(iii)' $\mathcal{S}_{\theta}$ is a dual grid;

(iv) $\mathcal{S}_{\theta}$ is a subGQ of $\mathcal{S}$ of order $\left(s^{\prime}, t^{\prime}\right), s^{\prime}, t^{\prime} \geq 2$.

Finally, we recall a result on fixed elements structures of whorls.

Theorem 2.5 ([27], 8.1.1). Let $\theta$ be a nontrivial whorl with center $x$ of the thick $G Q \mathcal{S}=$ $(\mathcal{P}, \mathcal{B}, I)$ of order $(s, t)$. Then one of the following must hold for the fixed element structure $\mathcal{S}_{\theta}=\left(\mathcal{P}_{\theta}, \mathcal{B}_{\theta}, \mathrm{I}_{\theta}\right)$.

(1) $y^{\theta} \neq$ y for each $y \in \mathcal{P} \backslash x^{\perp}$.

(2) There is a point $y, y \not x$, for which $y^{\theta}=y$. Put $V=\{x, y\}^{\perp}$ and $U=V^{\perp}$. Then $V \cup\{x, y\} \subseteq \mathcal{P}_{\theta} \subseteq V \cup U$, and $L \in \mathcal{B}_{\theta}$ if and only if $L$ joins a point of $V$ with a point of $U \cap \mathcal{P}_{\theta}$. 
(3) $\mathcal{S}_{\theta}$ is an ideal $\operatorname{subGQ}$ of order $\left(s^{\prime}, t\right)$, where $2 \leq s^{\prime} \leq s / t \leq t$, and hence $t<s$.

2.4. The dual net $\Pi(x)$. The following can be found in [26, 1.3.1]. Let $x$ be a regular point of a thick GQ $\mathcal{S}=(\mathcal{P}, \mathcal{B}, \mathrm{I})$ of order $(s, t)$. Then the incidence structure $\Pi(x)$ with

- POINT SET $x^{\perp} \backslash\{x\}$;

- LINE SET the set of spans $\{q, r\}^{\perp \perp}$, where $q$ and $r$ are noncollinear points of $x^{\perp} \backslash\{x\}$, and with the natural incidence, is the dual of a net of order $s$ and degree $t+1$. If in particular $s=t$, there arises a dual affine plane of order $s$. Also, in the case $s=t$, the incidence structure $\widehat{\Pi(x)}$ with point set $x^{\perp}$, with line set the set of spans $\{q, r\}^{\perp \perp}$, where $q$ and $r$ are different points in $x^{\perp}$, and with the natural incidence, is a projective plane of order $s$.

Theorem 2.6 ([38]). Suppose $\mathcal{S}=(\mathcal{P}, \mathcal{B}, I)$ is a thick $G Q$ of order $(s, t)$, with regular point $x$. Let $\mathcal{N}_{x}=\Pi(x)^{D}$ be the net which arises from $x$, and suppose $\mathcal{N}_{x}^{\prime}$ is a subnet of the same degree as $\mathcal{N}_{x}$. Then we have the following possibilities:

(1) $\mathcal{N}_{x}^{\prime}$ coincides with $\mathcal{N}_{x}$;

(2) $\mathcal{N}_{x}^{\prime}$ is an affine plane of order $t$ and $s=t^{2}$; also, from $\mathcal{N}_{x}^{\prime}$ there arises a proper subquadrangle of $\mathcal{S}$ of order $t$ having $x$ as a regular point.

If, conversely, $\mathcal{S}$ has a proper thick subquadrangle containing the point $x$ and of order $\left(s^{\prime}, t\right)$, then it is of order $t$, and hence $s=t^{2}$. Also, there arises a proper subnet of $\mathcal{N}_{x}$ which is an affine plane of order $t$.

2.5. Main Parameter Conjecture. The Main Parameter Conjecture (MPC) for finite generalized quadrangles (of order $(s, t), s \leq t)$ states that either $t=s$ ( $t$ a prime power), $t=s+2(t-1 \mathrm{a}$ prime power), $t=\sqrt[3]{s^{2}}$ ( $t$ a prime power) or $t=s^{2}$ ( $t$ a prime power). It is the analogue for finite generalized quadrangles of the Prime Power Conjecture for finite projective planes, but regarded to be even more out of reach. Many famous conjectures (such as Kantor's conjecture for EGQs or Payne's conjecture for STGQs) are special cases (even concentrating only on the prime power part, and forgetting about the precise form of $s$ and $t$ ).

There also exists a theory for the infinite case, and we refer to [45], and the references therein, for much more information on both the finite and infinite case.

We aim at establishing MPC for the entire class of STGQs in the present series of papers. 


\section{PART 0}

\section{SKEW TRANSLATION QUADRANGLES}

\section{STGQS}

For a generalized quadrangle $\mathcal{S}=(\mathcal{P}, \mathcal{B}, \mathrm{I})$, we call a point $x \in \mathcal{S}$ an elation point if there exists a group $K \leq \operatorname{Aut}(\mathcal{S})$ which fixes $x$ linewise and acts sharply transitively on $\mathcal{P} \backslash x^{\perp}$. We call the latter set the affine points w.r.t. $\mathcal{S}^{x}$. Similarly, the affine lines of $\mathcal{S}^{x}$ are the lines which are not incident with $x$. The group $K$ is the elation group of the elation generalized quadrangle (EGQ) $\left(\mathcal{S}^{x}, K\right)$. EGQs are the natural equivalents for generalized quadrangles as translation planes are for projective planes, although several basic questions for EGQs turn out to have different answers than in the theory for planes. For instance, elation groups are not necessarily abelian, and EGQs can have different (even non-isomorphic) elation groups for the same elation point.

Let $\left(\mathcal{S}^{x}, K\right)$ be a finite thick EGQ of order $(s, t)$. Let $z \in \mathcal{P}$ be not collinear with $x$, and let $\left\{U_{i} \mid i \in I\right\}$ be the lines incident with $z$, where for the index set $I$ we have $|I|=t+1$. For each $i \in I$, let $u_{i}:=\operatorname{proj}_{U_{i}} x$, and put $\left[U_{i}\right]:=x u_{i}$. Define $\mathcal{F}:=\left\{K_{U_{i}} \mid i \in I\right\}$ and $\mathcal{F}^{*}:=\left\{K_{u_{i}} \mid i \in I\right\}$, and note that choosing a different point for $z$, say $z^{\iota}$ with $\iota \in K$, just amounts to taking the image of each element of $\mathcal{F} \cup \mathcal{F}^{*}$ under $\iota$. So often we do not specify the point $z$ at all. For $A=K_{U_{j}} \in \mathcal{F}$, we denote $K_{u_{j}}$ by $A^{*}$ for the sake of convenience. Note the following properties:

(a) $|K|=s^{2} t$ and for all $A \in \mathcal{F}$ we have $|A|=s$ and $\left|A^{*}\right|=s t$; also, $|\mathcal{F}|=\left|\mathcal{F}^{*}\right|=|I|$;

(b) for all $A \in \mathcal{F}$ we have $A \leq A^{*}$;

(c) for all two by two different $A, B, C$ in $\mathcal{F}$ we have $A B \cap C=\{$ id $\}$;

(d) for different $A, B$ in $\mathcal{F}$ we have $A \cap B^{*}=\{$ id $\}$.

If an abstract group $K$ has the properties (a) through (d), we call $\left(\mathcal{F}, \mathcal{F}^{*}\right)$ a Kantor family of type $(s, t)$ in $K$. One then constructs an EGQ as follows: points are of three kinds - a symbol $(\infty)$ (which will be the elation point), cosets $k A^{*}$ for $k \in K$ and $A \in \mathcal{F}$ (the points collinear with and different from $(\infty)$ ), and the elements of $K$ (the affine points). Lines are of two kinds - symbols $[B]$ for $B \in \mathcal{F}$ (the lines on $(\infty)$ ) and cosets $r B$ for $r \in K$ and $B \in \mathcal{F}$ (the affine lines). Incidence is indicated by the descriptions we wrote between brackets. It is easy to show that this incidence structure $\Gamma\left(\mathcal{F}, \mathcal{F}^{*}\right)$ is an EGQ of order $(s, t)$, for which $(\infty)$ is the elation point and $K$ serves as elation group, acting by left multiplication. Moreover, starting from an EGQ $\left(\mathcal{S}^{x}, K\right)$ as above, one easily shows that $\left(\mathcal{S}^{x}, K\right)$ is isomorphic to $\left(\Gamma\left(\mathcal{F}, \mathcal{F}^{*}\right)^{(\infty)}, K\right)$.

Remark 3.1 (General case). Only slight adjustments are needed to incorporate the infinite case: in general, one replaces (a) by: (a) ${ }^{\prime}$ for all different $A, B \in \mathcal{F}$, we have that $A B^{*}=K$ and $K=A^{*} \cup\left(\cup_{C \in \mathcal{F}} A C\right)$.

3.1. Skew translation generalized quadrangles. A skew translation generalized quadrangle (STGQ) $\left(\mathcal{S}^{x}, K\right)$ is an EGQ with the additional local Moufang property that $K$ contains a subgroup $\mathbb{S}$ which consists of $t$ symmetries with center $x$ (which is the theoretical maximum). This property expresses precisely the fact that $K_{u, v}$ with $u \sim x \sim v \not u$ is independent of the choice of $u, v$ (all these groups coincide with $\mathbb{S}$ ). One can show that a finite EGQ $\left(\mathcal{S}^{x}, K\right)$ is an STGQ if and only if $x$ is a regular point. In terms of Kantor families, an EGQ is an STGQ if and only if for each $A, B \neq A$ in $\mathcal{F}, A^{*} \cap B^{*}$ is independent of the choice of $(A, B) \in \mathcal{F} \times \mathcal{F} \backslash$ diag. Another way to phrase this is asking that there is a normal subgroup $\mathbb{S}$ of $K$ such that for each $A \in \mathcal{F}$, $A^{*}=A \mathbb{S}$; for each $A, B(\neq A) \in \mathcal{F}$ we then have that $A^{*} \cap B^{*}=\mathbb{S}$. 
Up to a combination of Payne integration, translation duality and point-line duality, all known finite generalized quadrangles except the classical examples $\mathcal{H}\left(4, q^{2}\right)$ related to 4-dimensional projective Hermitian varieties and their duals are STGQs, and can hence be constructed from Kantor families of the type described in $\delta \S 3.1$.

No classification results are known for STGQs with general parameters; the only "general" result is the following, obtained independently by Chen [9] and Hachenberger [16].

Theorem $3.2([9,16])$. The parameters $s, t$ of a finite $S T G Q\left(\mathcal{S}^{x}, K\right)$ are powers of the same prime.

(Chen's proof was never published by him, but can be found in [47], along with many other results concerning EGQs and STGQs.) So $K$ is a $p$-group for some prime $p$. We often denote the prime $p$ by $\operatorname{char}\left(\mathcal{S}^{x}\right)$.

Before proceeding with describing the known STGQ constructions, we introduce general Heisenberg groups.

3.2. The general Heisenberg group. The general Heisenberg group $\mathcal{H}_{n}(q)$ of dimension $2 n+1$ over $\mathbb{F}_{q}$, with $n$ a natural number, is the group of square $(n+2) \times(n+2)$-matrices with entries in $\mathbb{F}_{q}$, of the following form (and with the usual matrix multiplication):

$$
\left(\begin{array}{ccc}
1 & \alpha & c \\
0 & \operatorname{id}_{n} & \beta^{T} \\
0 & 0 & 1
\end{array}\right)
$$

where $\alpha, \beta \in \mathbb{F}_{q}^{n}, c \in \mathbb{F}_{q}$ and with $\operatorname{id}_{n}$ being the $n \times n$-unit matrix. Let $\alpha, \alpha^{\prime}, \beta, \beta^{\prime} \in \mathbb{F}_{q}^{n}$ and $c, c^{\prime} \in \mathbb{F}_{q}$; then

$$
\left(\begin{array}{ccc}
1 & \alpha & c \\
0 & \operatorname{id}_{n} & \beta^{T} \\
0 & 0 & 1
\end{array}\right) \times\left(\begin{array}{ccc}
1 & \alpha^{\prime} & c^{\prime} \\
0 & \operatorname{id}_{n} & \beta^{\prime} \\
0 & 0 & 1
\end{array}\right)=\left(\begin{array}{ccc}
1 & \alpha+\alpha^{\prime} & c+c^{\prime}+\left\langle\alpha, \beta^{\prime}\right\rangle \\
0 & \operatorname{id}_{n} & \beta+\beta^{\prime} \\
0 & 0 & 1
\end{array}\right) .
$$

Here $\langle x, y\rangle$, with $x=\left(x_{1}, x_{2}, \ldots, x_{n}\right)$ and $y=\left(y_{1}, y_{2}, \ldots, y_{n}\right)$ elements of $\mathbb{F}_{q}^{n}$, denotes $x_{1} y_{1}+$ $x_{2} y_{2}+\cdots+x_{n} y_{n}=x y^{T}$. Note that $\mathcal{H}_{n}(q)$ is isomorphic to the group $\left\{(\alpha, c, \beta) \mid \alpha, \beta \in \mathbb{F}_{q}^{n}, c \in\right.$ $\left.\mathbb{F}_{q}\right\}$, where the group operation $\circ$ is given by

$$
(\alpha, c, \beta) \circ\left(\alpha^{\prime}, c^{\prime}, \beta^{\prime}\right)=\left(\alpha+\alpha^{\prime}, c+c^{\prime}+\alpha{\beta^{\prime}}^{T}, c+c^{\prime}\right) .
$$

Theorem 3.3 (see [47], chapter 4). The following properties hold for $\mathcal{H}_{n}(q)$ (defined over $\mathbb{F}_{q}$ ).

(i) $\mathcal{H}_{n}(q)$ has exponent $p$ if $q=p^{h}$ with $p$ an odd prime; it has exponent 4 if $q$ is even.

(ii) The center of $\mathcal{H}_{n}(q)$ is given by $Z\left(\mathcal{H}_{n}(q)\right)=\left\{(0, c, 0) \mid c \in \mathbb{F}_{q}\right\}$.

(iii) For each $(\alpha, c, \beta) \in \mathcal{H}_{n}(q)$, we have $(\alpha, c, \beta)^{-1}=\left(-\alpha,-c+\alpha \beta^{T},-\beta\right)$.

(iv) $\left[\mathcal{H}_{n}(q), \mathcal{H}_{n}(q)\right]=Z\left(\mathcal{H}_{n}(q)\right)$ and $\mathcal{H}_{n}(q)$ is nilpotent of class 2.

Define the following map

$$
\chi: V \times V \longmapsto Z\left(\mathcal{H}_{n}(q)\right):\left(a Z\left(\mathcal{H}_{n}(q)\right), b Z\left(\mathcal{H}_{n}(q)\right)\right) \longmapsto[a, b],
$$

where $V:=\mathcal{H}_{n}(q) / Z\left(\mathcal{H}_{n}(q)\right)$. Then $\chi$ is a BAN-form of $V$, which can be seen as a vector space over $\mathbb{F}_{q}$. If $n=1$, the polar space of $\chi$ (which is the natural incidence geometry of the absolute subspaces of $\chi)$ in the projective space $\mathbf{P}(V)$ coming from $V$ is a projective line $\mathbf{P G}(1, q)$; if $n=2$, a classical quadrangle $\mathcal{W}(q)$ arises. 
3.3. Examples. In this subsection, $\mathbf{P G}(n, q)$ - where $n$ is in $\mathbb{N} \cup\{-1\}$ and $q=p^{h}$ is a prime power - is the $n$-dimensional projective space over the field $\mathbb{F}_{q}$ (as a building). Its automorphism group is $\mathbf{P} \Gamma \mathbf{L}_{n+1}(q) \cong \mathbf{P G} \mathbf{L}_{n+1}(q) \rtimes \operatorname{Gal}\left(\mathbb{F}_{q} / \mathbb{F}_{p}\right)$.

We start with describing the known examples of finite STGQs, and make some side remarks on the way.

3.3.1. $\mathcal{W}(q), q$ odd. The points of $\mathbf{P G}(3, q)$ together with the totally isotropic lines with respect to a symplectic polarity form a GQ $\mathcal{W}(q)$ of order $q$ which is not self-dual. A symplectic polarity $\Theta$ of $\mathbf{P G}(3, q)$ has the following canonical form:

$$
X_{0} Y_{3}+X_{1} Y_{2}-X_{2} Y_{1}-X_{3} Y_{0}
$$

For each point $x, \mathcal{W}(q)^{x}$ is an STGQ w.r.t precisely one elation group, which is isomorphic to the 3-dimensional general Heisenberg group $\mathcal{H}_{1}(q)$ over $\mathbb{F}_{q}$.

No other STGQs of odd order $s$ are known.

3.3.2. TGQs of even order $q$. Each EGQ $\left(\mathcal{S}^{x}, K\right)$ of even square order $(q, q)$ for which the elation group is abelian (in which case one calls the EGQ also a translation generalized quadrangle) is also an STGQ. (In that case, $K$ is elementary abelian.) Many such examples are known, cf. [37].

All known even square order STGQs are in fact TGQs.

3.3.3. Hermitian quadrangles $\mathcal{H}\left(3, q^{2}\right)$. Next, let $\mathcal{H}$ be a nonsingular Hermitian variety in $\mathbf{P G}\left(3, q^{2}\right)$. The $\mathbb{F}_{q}$-rational points and lines of $\mathcal{H}$ form a generalized quadrangle $\mathcal{H}\left(3, q^{2}\right)$, which has order $\left(q^{2}, q\right)$. The variety $\mathcal{H}$ has the following canonical form:

$$
X_{0}^{q+1}+X_{1}^{q+1}+X_{2}^{q+1}+X_{3}^{q+1}=0 .
$$

For each point $x, \mathcal{H}\left(3, q^{2}\right)^{x}$ is an STGQ w.r.t. an elation group which is isomorphic to the 5dimensional general Heisenberg group $\mathcal{H}_{2}(q)$ over $\mathbb{F}_{q}$. If $q$ is odd, there is precisely one elation group per point; if $q$ is even, there exists a second isomorphism class of elation groups, as observed by Rostermundt [30] and, independently, the author [42].

3.3.4. Generalization: $\mathcal{H}\left(3, q^{2}\right) \mapsto$ flock quadrangles. A flock $\mathcal{F}$ of the quadratic cone $\mathcal{K}$ in $\mathbf{P G}(3, q)$ is a partition of $\mathcal{K}$ without its vertex into $q$ irreducible conics. Choosing coordinates in such a way that the equation for $\mathcal{K}$ becomes $X_{0} X_{1}=X_{2}^{2}$, one can show that the equations of the conic planes of the flock define a certain Kantor family in $\mathcal{H}_{2}(q)$ of type $\left(q^{2}, q\right)$, and the associated quadrangle is a flock quadrangle [34]. It is often denoted as $\mathcal{S}(\mathcal{F})$. If one chooses a special flock for which all conic planes share some exterior line to the flock, one can show that $\mathcal{S}(\mathcal{F}) \cong \mathcal{H}\left(3, q^{2}\right)$.

All flock quadrangles are STGQs with elation group $\mathcal{H}_{2}(q)$, and this class of GQs was the main motivation for Payne to consider and study the MSTGQs in his celebrated paper [24] (cf. the next section).

3.3.5. Dual Suzuki-Tits quadrangles. This class and its Kantor family is described in detail in the next section.

3.3.6. Translation duals of suitable GQs. Let $n, m$ be nonzero positive integers. An egg $\mathcal{O}=$ $\mathcal{O}(n, m, q)$ is a certain set of $(n-1)$-spaces in $\mathbf{P G}(2 n+m-1, q)$ having tangent spaces at each of its elements, as such generalizing ovoids of $\mathbf{P G}(3, q)$ in a natural way, see [37]. Similarly as for ovoids, one can construct TGQs $\mathbf{T}(\mathcal{O})$ from eggs and conversely, it can be shown that each TGQ is isomorphic to a $\mathbf{T}(\mathcal{O})$. This natural generalization of Tits quadrangles has led to examples of quadrangles which weren't covered by the Tits construction. (We refer to [37] for all the details.) 
Now let $n \neq m$. The tangent spaces of an egg $\mathcal{O}(n, m, q)$ in $\mathbf{P G}(2 n+m-1, q)$ form an egg $\mathcal{O}^{*}(n, m, q)$ in the dual space of $\mathbf{P G}(2 n+m-1, q)$. So in addition to the TGQ $\mathbf{T}(\mathcal{O})$, a TGQ $\mathbf{T}\left(\mathcal{O}^{*}\right)$ arises which is not necessarily isomorphic to $\mathbf{T}(\mathcal{O})$. The egg $\mathcal{O}^{*}(n, m, q)=\mathcal{O}^{*}$ will be called the translation dual of $\mathcal{O}(n, m, q)=\mathcal{O}$, and $\mathbf{T}\left(\mathcal{O}^{*}\right)$ will be called the translation dual of $\mathbf{T}(\mathcal{O})$.

The most studied class of eggs in odd characteristic is the class of eggs which are "good" at some element, a property which is satisfied for each point of any ovoid, and which expresses the fact that the corresponding "good TGQ" has many full subGQs on the line corresponding to the good element. In that case, $m=2 n$, and moreover, the point-line dual of $\mathbf{T}\left(\mathcal{O}^{*}\right)$ is isomorphic to a flock quadrangle.

$$
\text { good TGQ } \mathbf{T}(\mathcal{O}) \stackrel{*}{\longmapsto} \text { translation dual } \mathbf{T}\left(\mathcal{O}^{*}\right) \stackrel{D}{\longmapsto} \text { flock quadrangle }
$$

In [43], solving a question of Kantor affirmatively, and using the machinery of [40, 39, 41], the author showed that this construction produced a new class of STGQs, namely the point-line duals $\mathbf{T}(\mathcal{O})^{D}$ of $\mathbf{T}(\mathcal{O})$. Moreover, the author later showed that if $\mathbf{T}(\mathcal{O}) \nRightarrow \mathcal{H}\left(3, q^{2}\right)$, the corresponding (unique) elation groups were new, not isomorphic to $\mathcal{H}_{2}(q)$, as a direct corollary of the next theorem.

Theorem $3.4([48])$. Let $\mathcal{S}$ be an EGQ of order $\left(q^{2}, q\right)$, $q$ any prime power, with elation group $\mathcal{H}_{2}(q)$. Then $\mathcal{S}$ is a flock quadrangle.

3.4. Central STGQs. Each of the examples described above has the property that the group of symmetries $\mathbb{S}$ with center the elation point of the STGQ is a central subgroup of the elation group. In general, we call STGQs with this property central STGQs. As we will see in the next section, the property of being central is equivalent to the strong local Moufang property "(MSTGQ1) $)^{b}$," and allows one to control a great number of properties of STGQs. The latter claim will become clear throughout the present series of papers.

3.5. Infinite case. In the infinite case, there are other interesting examples - see for instance Knarr [22]. 


\section{PART I}

\section{STRUCTURE THEORY}

\section{LOCAL MOUfANG CONDITIONS}

Before proceeding, we need the condition " $(M)_{x}$." Let $\mathcal{S}$ be a GQ, and let $x$ be a point of $\mathcal{S}$. Then $\mathcal{S}$ satisfies $(M)_{x}$ if for any i-root $(x, L, y)(x \mathrm{I} L \mathrm{I} y \neq x)$, and any line $C \mathrm{I} y, C \neq L$, the automorphism group of $\mathcal{S}$ that fixes $x$ and $y$ linewise, and $L$ pointwise, $\operatorname{Aut}(\mathcal{S})_{[\{x, L, y\}]}$, acts transitively on the points of $C \backslash\{y\}$. In that case, the action is sharply transitive, and the definition is independent of $C$ (or $y$ ). So $(M)_{x}$ holds if every i-root on $x$ is Moufang (by definition).

4.1. MSTGQs. Now let $\left(\mathcal{S}^{x}, \ell\right)$ be a finite EGQ with the following additionial properties:

(MSTGQ1) $\mathcal{S}^{x}$ satisfies property $(M)_{x}$;

(MSTGQ2) for each point $y \sim x \neq y, \ell_{[\{x, x y, y\}]}=: \ell(y)$ has the property that if $\alpha \in \ell(y)^{\times}$fixes some affine line $U$ (which must be concurrent with $x y$ as $\alpha \neq \mathrm{id}$ ), it also fixes $U \cap x y$ linewise;

(MSTGQ3) no line $U \mathrm{I} x$ is the unique center of a triad $\{V, W, X\}$, where $V \mathrm{I} x$.

Payne introduced such EGQs in [24], and calls them Moufang skew translation generalized quadrangles (MSTGQs). He shows in loc. cit. that the point $x$ is regular, making it a center of symmetry for a subgroup $\mathbb{S} \leq \ell$, and $\mathbb{S} \leq Z(\ell)$ is elementary abelian. Moreover, for any $u \sim x \neq u, \ell(u)$ is also elementary abelian, and $\ell$ is a $p$-group. So $\mathcal{S}^{x}$ is an STGQ, but it is of a very particular type: not all STGQs satisfy (MSTGQ1) (as we will see in $\S \$ 4.4$ ), and no known square STGQ of even order has (MSTGQ3). A more general version of (MSTGQ1) seems to be true (now supposing that $\left(\mathcal{S}^{x}, \ell\right)$ is an STGQ), namely:

$(\text { MSTGQ1) })^{b}$ Each $\alpha \in \ell^{\times}$which fixes some affine line $U$, also fixes $U \cap \operatorname{proj}_{U} x$ linewise.

Already from (MSTGQ1) ${ }^{b}$, one can prove that the symmetry group $\mathbb{S}$ is in the center of $\ell$, and conversely, $\mathbb{S} \leq Z(\ell)$ implies (MSTGQ1) ${ }^{b}$. So this seems the quintessential Moufang property for STGQs to study and obtain.

4.2. Property (G). In this subsection we have a deeper look into the so-called "Property (G)."

Let $x$ be a point of a GQ $\mathcal{S}$ of order $\left(t^{2}, t\right)$, and let $U, V$ be distinct lines incident with $x$. Then $\mathcal{S}$ satisfies Property $(G)$ at the pair $\{U, V\}$ if any triad of lines $\{V, W, Z\}$ in $U^{\perp}$ is 3-regular (which means that $\left|\{V, W, Z\}^{\perp}\right|=\left|\{V, W, Z\}^{\perp \perp}\right|=t+1$. (Note that the definition is symmetric in $U$ and $V$.) The flag $(x, L)$ has Property $(G)$ if all pairs of distinct lines $\{L, M\}$ "on $x$ " have $(\mathrm{G})$. One says that $x$ has Property $(G)$ if all pairs $\{U, V\}$ "on $x$ " have Property $(\mathrm{G})$.

The following theorem was first obtained in odd characteristic in [35], answering a fundamental conjecture of Payne's essay [24]. In the case of even characteristic, it was obtained only much later by M. R. Brown [8]. (In odd characteristic, only one flag was required in [35].)

Theorem 4.1 ([8, 35]). A GQ of order $\left(t^{2}, t\right)$ satisfying Property $(G)$ at two distinct flags $(u, L)$ and $(u, M)$ for a point $u$ is isomorphic to a flock $G Q$.

Corollary 4.2. A GQS of order $\left(t^{2}, t\right)$ with $t$ even is a flock $G Q$ if and only if it contains a point $x$ which is in $t^{3}+t^{2}$ distinct subTGQs of order $t$ for which $x$ is a translation point.

Proof. It is well known that $t^{3}+t^{2}$ is the maximum number of subGQs of order $t$ that contain the same point in a GQ of order $\left(t^{2}, t\right)$. If this is the case for $x$, we have that for any triad of lines $\{U, V, W\}$ for which $U I x$ and which has a center $C$ which is incident with $x, U, V, W, C$ and $D$ are contained in a subGQ $\mathcal{S}^{\prime}$ of order $t$, where $D$ is any center of $\{U, V, W\}$ different from $C$. 
Since $\mathcal{S}^{\prime}$ is a TGQ with translation point $x$, any line incident with $x$ is regular in $\mathcal{S}^{\prime}$, and so it easily follows that $x$ satisfies Property (G). Whence by Theorem 4.1, $S$ is a flock GQ.

Conversely, it is well known that a flock GQ of order $\left(t^{2}, t\right)$ with $t$ even has $t^{3}+t^{2}$ subTGQs of order $t$ containing the special point, which is a common translation point for all these subGQs.

Let $\mathcal{S}$ be a GQ satisfying the same properties as in the statement of Corollary 4.2, but now with $t$ odd. Then by the same reasoning, $\mathcal{S}$ is a flock GQ. The only flock GQs in odd characteristic with ideal subGQs are isomorphic to the Kantor-Knuth flock quadrangles [37], and in that case, all ideal subGQs are isomorphic to $\mathcal{W}(t)$, contradicting the fact that we assumed the subGQs to be TGQs (when $t$ is odd, $\mathcal{W}(t)$ is not a TGQ since it does not contain regular lines). So for $t$ odd, Corollary 4.2 makes no sense.

4.3. The Suzuki-Tits quadrangles. Let $q=2^{e}$ be an odd power of 2 , and let $\sigma \in \operatorname{Aut}\left(\mathbb{F}_{q}\right)$ be such that $\sigma^{2}=2$ (so that $\sigma$ is by definition a Tits endomorphism). Now define a map

$$
f: \mathbb{F}_{q}^{2} \longmapsto \mathbb{F}_{q}^{2}:(a, b) \longmapsto a^{\sigma+2}+a b+b^{\sigma} .
$$

The Tits ovoid of $\mathbf{P G}(3, q)$ is then given by

$$
\mathcal{O}=\{(0,1,0,0)\} \cup\left\{(1, f(a, b), a, b) \mid a, b \in \mathbb{F}_{q}\right\} .
$$

It is well known that this ovoid admits the natural 2-transitive action of the Suzuki group $\mathbf{S z}(q)$. Embed $\mathbf{P G}(3, q)$ as a hyperplane in $\mathbf{P G}(4, q)$ by $(x, y, z, w) \longmapsto(0, x, y, z, w)$. Construct the TGQ $\Gamma=\mathbf{T}_{3}(\mathcal{O})$, and call it the Suzuki-Tits quadrangle. Then $\operatorname{Aut}(\Gamma)_{(\infty)}$ (where $(\infty)$ is the special point of $\mathbf{T}_{3}(\mathcal{O})$ ) admits the natural 2-transitive action of $\mathbf{S z}(q)$ on the $q^{2}+1$ lines incident with $x$. Also, $\mathbf{T}_{3}(\mathcal{O})$ is classical if and only if $\mathcal{O}$ is an ellipic quadric if and only if $q=2$.

Let $[a, b, c, d, e]$ with $a, b, c, d, e \in \mathbb{F}_{q}$ be defined as follows:

$$
[a, b, c, d, e]=\left(\begin{array}{ccccc}
1 & 0 & c & d & e \\
0 & 1 & f(a, b) & a & b \\
0 & 0 & 1 & 0 & 0 \\
0 & 0 & a^{\sigma+1}+b & 1 & a^{\sigma} \\
0 & 0 & a & 0 & 1
\end{array}\right) .
$$

Now define collineations $\theta(a, b, c, d, e):(u, x, y, z, w) \longmapsto(u, x, y, z, w)[a, b, c, d, e]$ of $\mathbf{P G}(4, q)$. Then by [25], $G=\left\{\theta(a, b, c, d, e) \mid a, b, c, d, e \in \mathbb{F}_{q}\right\}$ is a group of order $q^{5}$ with binary operation

$$
\begin{aligned}
& {[a, b, c, d, e]\left[a^{\prime}, b^{\prime}, c^{\prime}, d^{\prime}, e^{\prime}\right]} \\
& =\left[a+a^{\prime}, b+b^{\prime}+a a^{\prime \sigma}, c+c^{\prime}+d\left(a^{\prime \sigma+1}+b^{\prime}\right)+e a^{\prime}, d+d^{\prime}, e+e^{\prime}+d a^{\prime \sigma}\right] .
\end{aligned}
$$

The group $G$ leaves $\mathcal{O}$ invariant; it fixes the line $L$ of $\Gamma$ corresponding to $(0,0,1,0,0) \in \mathcal{O}$ pointwise, and acts sharply transitively on the lines not concurrent with $L$. So $\Gamma^{D}$ is an EGQ with elation group $G$. The center of $G$ is $Z(G)=\left\{[0,0, c, 0,0] \mid c \in \mathbb{F}_{q}\right\}$, and is a group of symmetries about $L$. So the dual of $\Gamma$ is a central STGQ of order $\left(t^{2}, t\right)$.

Now define for $t \in \mathbb{F}_{q}$ :

$$
A(t)=\left\{[a, b, t f(a, b), t a, t b] \mid a, b \in \mathbb{F}_{q}\right\}, A^{*}(t)=\left\{[a, b, c, t a, t b] \mid a, b, c \in \mathbb{F}_{q}\right\},
$$

and also put

$$
A(\infty)=\left\{[0,0,0, d, e] \mid d, e \in \mathbb{F}_{q}\right\}, A^{*}(\infty)=\left\{[0,0, c, d, e] \mid c, d, e \in \mathbb{F}_{q}\right\} .
$$


Then $\mathcal{F}=\left\{A(t) \mid t \in \mathbb{F}_{q} \cup\{(\infty)\}\right\}$ and $\mathcal{F}^{*}=\left\{A^{*}(t) \mid t \in \mathbb{F}_{q} \cup\{(\infty)\}\right\}$ define the Kantor family of $G$ corresponding to the point $\langle(1,0,0,0,0),(0,1,0,0,0)\rangle$ of $\Gamma^{D}$.

4.4. Some strange properties. The following structure lemma is easy, but rather mysterious due to (ii), (iii) and (vi), cf. the paragraph after the observation. Below, $\Gamma=\mathbf{T}_{3}(\mathcal{O})$ with $\mathcal{O}$ a SuzukiTits ovoid; $G$ is the group defined in the previous subsection, and it acts as an elation group for $\Gamma^{D}$.

Observation 4.3. $\quad$ (i) $Z(G)$ is the symmetry group w.r.t. the elation point, and is elementary abelian.

(ii) $A^{*}(\infty)$ and $A(\infty)$ are elementary abelian and $A^{*}(\infty) \unlhd G$, so that $A^{*}(\infty)$ fixes $[A(\infty)]$ pointwise.

(iii) For $t \in \mathbb{F}_{q}, A^{*}(t)$ and $A(t)$ are non-abelian of exponent 4 ; moreover, for $t \neq t^{\prime}, A^{*}(t) \cong$ $A^{*}\left(t^{\prime}\right)$ and $A(t) \cong A\left(t^{\prime}\right)$. Also, no $A^{*}(t)$ is normal in $G$ and hence no $A^{*}(t)$ does fix $[A(t)]$ pointwise.

(iv) $G$ is nonabelian of exponent 4 and $G / Z(G)$ is not abelian.

(v) $G$ is the complete set of elations about $x$.

(vi) $\Gamma^{D}$ is not an $M S T G Q$.

(vii) $[G, G]$ is strictly contained in $A^{*}(\infty)$.

Proof. (i)-(ii)-(iii) are obvious. The fact that $G / Z(G)$ is not abelian follows from the fact that for any $t \in \mathbb{F}_{q}, A^{*}(t) / Z(G) \cong A(t)$. Claim (v) is proved in [28]. Claim (vi) follows from (v) and (iii). Finally, clearly $[G, G]$ fixes $[A(\infty)]$ pointwise so that it is a subgroup of $A^{*}(\infty)$. As $G / A^{*}(\infty)$ is isomorphic to $A(t)$ for any $t \in \mathbb{F}_{q}$, it follows that $A^{*}(\infty) \neq[G, G]$ by (iii).

Note that by (ii) and (iii), each i-root $(x,[A(\infty)], z)$ is Moufang, while no other i-root of $\Gamma^{D}$ is.

The Kantor family described in the previous subsection is, by (ii)-(iii), the only known one for which not all elements are isomorphic. Besides that, $\Gamma^{D}$ is the only known STGQ which is not an MSTGQ. The elation group $G$ can not be constructed by the twisting method described in this paper - essentially there are two reasons; first of all, the fact that $\Gamma^{D}$ is not an MSTGQ (which is needed for making the twist), and secondly, if $\Gamma^{D} \neq \mathcal{H}\left(3, t^{2}\right)$, it cannot contain subGQs of order $t$ which are fixed elementwise by some involution of $\mathbf{T}_{3}(\mathcal{O})$ (and such involutions are also needed).

Observation 4.4. Let $[\infty]$ be the special line of $\Gamma^{D}$ corresponding to $\mathcal{O}$. Then every point incident with $[\infty]$ is an elation point for a unique elation group.

Proof. Follows from Observation 4.3 v) and the fact that $\operatorname{Aut}(\Gamma)^{D}$ is transitive on the lines through $x$.

4.5. SubTGQs of $\Gamma^{D}$. We keep working with $\Gamma$ and $\mathcal{O}$ as in $\S \$ 4.4$.

Each nontangent plane section $\Pi \cap \mathcal{O}=\mathcal{O}^{\prime}$ defines a $\mathbf{T}_{2}\left(\mathcal{O}^{\prime}\right)$ of Tits, and hence a full subTGQ of $\mathbf{T}_{3}(\mathcal{O})$. Moreover, any such $\mathcal{O}^{\prime}$ is a translation oval, so that $\mathbf{T}_{2}\left(\mathcal{O}^{\prime}\right)^{D}$ also is a subTGQ of $\Gamma^{D}$. Whence for any choice of $x \in \mathcal{O}, \Gamma^{D}$ admits precisely $q^{3}+q^{2}$ subTGQs of order $q$ that contain $x$. Still, $\Gamma^{D}$ is not a flock GQ (supposing that it is not classical). The reason why Corollary 4.2 cannot be applied is reflected in the following theorem.

Theorem 4.5. Let $\mathcal{O}$ be non-classical.

(i) Then $\Gamma^{D}$ contains precisely $q^{4}+q^{2}$ distinct subTGQs of order $q$ which all contain $[\infty]$, and for which the (unique) translation point is a point of $[\infty]$.

(ii) Each point $z \mathrm{I}[\infty]$ is contained in precisely $q^{3}+q^{2}$ subGQs of order $q$. Precisely $q^{2}$ of them have $z$ as (unique) translation point. For each other point $z^{\prime} \mathrm{I}[\infty], z^{\prime} \neq z$, there are precisely $q$ subGQs of order $q$ containing $\left\{z, z^{\prime}\right\}$, and having $z^{\prime}$ as (unique) translation point. 
(The proof is left to the reader.)

\section{FIX POINT THEORY AND STRUCTURE}

In this section, $\left(\mathcal{S}^{x}, G\right)$ is an STGQ of order $(s, t)$ with $\mathbb{S}$ the associated group of symmetries about $x$.

5.1. Property (*). We need to introduce the following crucial property:

(*) If $y \sim x \neq y$ is fixed by the element $g$ of $G$, then $g$ fixes $y x$ pointwise. In other words, for each $U I x$, let $N_{U}$ be the kernel of the action of $G$ on the points of $U$; then $G / N_{U}$ acts freely on $U \backslash\{x\}$.

We will also say that $(*)$ is satisfied for a subgroup $A \leq G$ if the property holds restricted to $A$. Also, we say that an EGQ $\left(\mathcal{S}^{x}, G\right)$ satisfies $(*)$ if $G$ does.

Theorem 5.1. An STGQ satisfies (*) if and only if $T=G / \mathbb{S}$ is abelian. If one of these equivalent conditions is satisfied, the members of $\mathcal{F}$ are abelian.

Proof. If $T$ is abelian, $\mathcal{S}^{x}$ obviously satisfies (*), as $G$ acts transitively on the point sets $L \backslash\{x\}$, $L I x$. Now let $\mathcal{S}^{x}$ satisfy $(*)$. Consider the associated Kantor family $\left(\mathcal{F}, \mathcal{F}^{*}\right)$, and let $A \in \mathcal{F}$. Define $G_{A}=\langle B \mid B \in \mathcal{F} \backslash\{A\}\rangle$, and note that $G=G_{A}$. Note also that for $a \in A$ and $c \in C$, $C \in \mathcal{F} \backslash\{A\}$, we have that $[a, c] \in \mathbb{S}$. So $\left[A, G_{A}\right]=[A, G]$ vanishes in $T$. It now easily follows that $T$ is abelian. As for the final part of the statement, note that for $A \in \mathcal{F}, A \cong A \mathbb{S} / \mathbb{S}$.

Later, we will study STGQs for which all members of $\mathcal{F}$ are abelian, but $G / \mathbb{S}$ is not.

\subsection{Fixed point theory.}

Theorem 5.2 (Fix point structure for STGQs). Let $\mathcal{S}=\left(\mathcal{S}^{x}, G\right)$ be an $S T G Q$ of order $(s, t)$. For fix $(e)$, e being an element of $G^{\times}$, we have the following possibilities.

(i) $\operatorname{fix}(e)=x^{\perp}$;

(ii) $\operatorname{fix}(e)=\{x\}$;

(iii) $\operatorname{fix}(e)$ is a subset of some line on $x$ which is different from $\{x\}$;

(iv) If we are in (iii), and $\alpha(e):=|\operatorname{fix}(e)|-1$ is a power of $p:=\operatorname{char}\left(\mathcal{S}^{x}\right)$, then e fixes affine lines if $s$ is not even when $s=t$;

(v) If $s=t$, G satisfies Property (*);

(vi) If $s=t$, e always has a fixed point different from $x$ (that is, any element of $H$ either fixes one or all lines on $x$ pointwise);

(vii) If $K(e)$ is the set of fixed affine lines of e, then $|K(e)| \equiv 0 \bmod p$.

Proof. If we are not in case (ii) and case (iii), so that $e$ has fixed points $y, z \nsim y$, then obviously $e$ must be a symmetry about $x-$ i.e., fix $(e)=x^{\perp}$.

Consider case (iv). Suppose that $e$ does not fix affine lines, and suppose that $s$ is odd when $s=t$. We want to apply [26, 1.9.1] (a result first obtained by Benson), which states that for any automorphism $\beta$ of a GQ of order $(s, t)$, one has

$$
(t+1)|\operatorname{fix}(\beta)|+g(\beta) \equiv s t+1 \quad \bmod s+t, \quad[\text { Benson's identity }]
$$

where $g(\beta)$ is the number of points that are mapped onto a collinear but different point under $\beta$. Hence we have

$$
(t+1)|\operatorname{fix}(e)|+t s+(s+1-|\operatorname{fix}(e)|) \equiv s t+1 \quad \bmod s+t
$$


or also

$$
t \alpha(e) \equiv 0 \bmod s+t,
$$

contradiction. So (iv) follows from (iii).

(v) follows immediately from the plane structure of $\Gamma(x)$, as does (vi).

Finally, let $K(e)$ be as in (vii) (supposing that $K(e) \neq \emptyset$, as the empty case is trivial). Then $C_{G}(e)$ is nontrivial and it is easy to find prime elements in $C_{G}(e)$ (for instance in $C_{\mathbb{S}}(e)$ ) which act without trivial orbits on $K(e)$. So $|K(e)| \equiv 0 \bmod p$.

Remark 5.3. Note that all these possibilities do occur.

Let $\left(\mathcal{S}^{x}, G\right)$ be an STGQ of order $(s, t)$. Let $z \in Z=Z(G)$ be a central element, and suppose $z$ fixes some line $M \mp x$; then clearly any line meeting $\operatorname{proj}_{x} M$ is fixed by $z$. Plugging this into Benson's identity (for points), one obtains

$$
(t+1)(s+1)+(s+1) s t \equiv s t+1 \bmod s+t .
$$

On the other hand, since $\mathcal{S}^{x}$ admits point-symmetries, we have the dual

$$
(t+1) s t \equiv 0 \quad \bmod s+t,
$$

so that $s / t+1$ divides $\operatorname{gcd}(s+1, t+1)$ if $t \neq s$. In this (for our means for now only interesting) latter case, we can put $s=p^{n(a+1)}$ and $t=p^{n a}, p$ being a prime and $n, a$ natural numbers. As for $c \in \mathbb{N}_{0,1}, c^{n}+1$ divides $c^{m}+1$ if and only if $m \equiv 0 \bmod n$ and $m / n$ is odd, we have a contradiction. So we have:

Lemma 5.4. (i) If $s \neq t$ no element of $Z^{\times}$sends an affine point to a collinear point. In particular, if the conditions of Theorem 5.2 (iv) are also satisfied, elements of $Z^{\times}$either fix only the point $x$, or are contained in $\mathbb{S}$.

(ii) If $s \neq t, \mathcal{S}^{x}$ does not admit line symmetries.

Now let $N I x$, and $M \sim N, M \mp x$. Let $g \in G_{M}^{\times}, \alpha$ the number of fixed points on $N$ besides $x$, and $f$ the number of fixed affine lines. Then Benson's identity for lines together with Lemma 5.4(i) tells us that

$$
(s+1)(t+1+f)+\alpha t-f \equiv s t+1 \bmod s+t,
$$

so that

$$
\alpha-f \equiv 0 \quad \bmod s / t+1
$$

First note that $f$ is a power of $p$ if $(*)$ is satisfied. First let $y \mathrm{I} N$ be a point different from $x$ which is incident with precisely $f_{y}>0$ fixed affine lines for $\alpha$; clearly $f_{y}>1$. Let $\mathcal{L}_{y}$ be the set of fixed affine lines on $y$. Then any element of $\mathbb{S}$ which stabilizes $\mathcal{L}_{y}$ obviously centralizes $\alpha$, so that $C_{\mathbb{S}}(\alpha)$ acts sharply transitively on $\mathcal{L}_{y}$. Whence the latter set's order is a power of $p$. This observation holds in general, that is, without assuming $(*)$, so we state it as an intermediate observation:

Observation 5.5. Let $\left(\mathcal{S}^{x}, G\right)$ be an $S T G Q$, and let $\alpha \in G$ fix some affine line incident with $y \sim x \neq y$. Let $\mathcal{L}_{y}$ be the set of fixed affine lines on $y$. Then $C_{\mathbb{S}}(\alpha)$ acts sharply transitively on $\mathcal{L}_{y}$. In particular, if the $S T G Q$ is finite, $\left|\mathcal{L}_{y}\right|=\left|C_{\mathbb{S}}(\alpha)\right|$. 
Proceeding as before the observation, if $U$ and $V$ are distinct fixed affine lines for $\alpha, U \not V$, any element $\beta$ of $G$ sending $U$ to $V$ centralizes $\alpha$, as $[\alpha, \beta]$ fixes $V$ and is contained in $\mathbb{S}$ by (*).

5.3. Averaging lemma. The following lemma will be used many times.

Lemma 5.6 (Averaging lemma). Let $\left(\mathcal{S}^{x}, G\right)$ be an $S T G Q$ of order $(s, t)$ with $(*)$, where $s \neq t$ if $s$ is even. Let $L$ be an affine line. Then each element of $G_{L}^{\times}$fixes precisely s affine lines (and so $t+s+1$ lines in total). As a corollary, $f(\gamma) / \alpha(\gamma)$ is independent of the choice of $\gamma$ if $f(\gamma) \neq 0$. (In the case $s=t$ odd the latter condition is redundant.)

Proof. First let $s=t$ be odd. Let $\alpha \in G_{L}^{\times}$fix $f$ affine lines. As (*) holds, we have that

$$
\left|\alpha^{G}\right| \leq|[G, G]| \leq|\mathbb{S}|=s
$$

so that $\left|C_{G}(\alpha)\right| \geq s^{2}$. Let $\mathcal{P}_{\alpha}$ be the total set of affine points which are on affine lines of $\mathcal{S}^{x}$ fixed by $\alpha$. As $\left|C_{G}(\alpha)\right|=\left|\mathcal{P}_{\alpha}\right|$ by earlier considerations, it follows that $f \geq s$. This was already obtained for the other parameters under consideration. (Note that the same reasoning can be used to prove it in an alternative fashion for these parameters.)

Let $\gamma \in G_{L}^{\times}$; we know that $\gamma$ fixes precisely $s p^{e(\gamma)}$ affine lines, for some natural number $e(\gamma), p$ being $\operatorname{char}\left(\mathcal{S}^{x}\right)$. Let $X$ be the set of lines in $\operatorname{proj}_{x} L^{\perp}$ which are not incident with $x$. Since $G$ does not contain line symmetries, $G$ acts faithfully on $X$. Of course this action is transitive, so that the average number of fixed lines in $X$ of an element of $G$ is 1 . By (*), the elements of $G$ fixing elements of $X$ precisely are those of $G_{u} \backslash \mathbb{S}$, with $u$ an arbitrary (but "fixed") point of $\operatorname{proj}_{x} L \backslash\{x\}$. ( $G_{u}$ has size $s t$ and fixes $\operatorname{proj}_{x} L$ pointwise.) Let $s p^{\bar{e}}$ be the average number of elements of $X$ fixed by elements of $G_{u}^{\times}$. Then by the Orbit-Counting Lemma (on the permutation group $(G, X)$ ) we have

$$
1=\left((s t-t) s p^{\bar{e}}+s t\right) \frac{1}{s^{2} t},
$$

so that $\bar{e}=0$, and hence each element of $G_{u}^{\times}$fixes precisely $s$ affine lines.

Remark 5.7. The Averaging Lemma will provide us much combinatorial information once we are ready to pin down the isomorphism classes of STGQs.

The following corollary is important for many purposes.

Corollary 5.8. Let $\left(\mathcal{S}^{x}, G\right)$ be as in Lemma 5.6 Then $\left|C_{G}(\gamma)\right|=s^{2}$, so that $[\gamma, G]=\mathbb{S}$, for $\gamma \in\left(\cup_{u \in x^{\perp} \backslash\{x\}} G_{u}\right) \backslash \mathbb{S}$.

Proof. Let $\gamma$ be as in the statement. Then $\gamma$ fixes precisely $s$ affine lines by Theorem 5.2(iv) and the Averaging Lemma. Call the set of these lines $\mathcal{X}$. If $\beta \in G$ sends some line $M \in X$ to $M^{\beta} \in \mathcal{X}$, then clearly $[\gamma, \beta]=\mathrm{id}$. And to obtain the latter property, $\beta$ must stabilize $\mathcal{X}$. Besides that, (*) imposes the fact that $G_{L}$ is abelian for any affine $L$, and whence if $U \pm x$ is such that $\gamma \in G_{U}$ (such $U$ exists), $G_{U} \leq C_{G}(\gamma)$. The corollary easily follows from the fact that $G$ is transitive on $x$.

5.4. Transfer. The next theorem is formulated in terms of EGQs, and is quite general.

Theorem 5.9 (Transfer). Let $\left(\mathcal{S}^{x}, G\right)$ be an EGQ of order $(s, t)$, with $s>t$, and suppose that st is a power of the prime $p$. Let a $\in G^{\times}$be such that a fixes only points of some line $L_{a} I x$, and suppose that $\alpha(a)$, where $\alpha(a)+1$ is the number of fixed points of a on $L_{a}$, is a power of $p=\operatorname{char}\left(\mathcal{S}^{x}\right)$.

(i) Then $f(a)$, the number of fixed affine lines, is not zero. 
(ii) Suppose $f(a)$ is also a power of $p$. Then

$$
\frac{\alpha(a)}{f(a)}=\left(\frac{s}{t}\right)^{o(a)}
$$

where $o(a) \in 2 \mathbb{Z}$.

Proof. Applying Benson's identity (for points) on $a$, we obtain

$$
(t+1)|\operatorname{fix}(a)|+s t+f(a) s+(s+1-|\operatorname{fix}(a)|) \equiv s t+1 \bmod s+t,
$$

from which we deduce that

$$
\alpha(a)-f(a) \equiv 0 \bmod \frac{s}{t}+1
$$

Part (i) follows.

From now on, we suppose that $f(a)$ is also a power of $p$.

Let $f(a) \leq \alpha(a)$; as both are powers of $p$ by assumption, $f(a)$ divides $\alpha(a)$. We conclude that

$$
\frac{\alpha(a)}{f(a)}-1 \equiv 0 \quad \bmod \frac{s}{t}+1
$$

It is well known that for positive integers $c>1, m>1, n>1$, we have that $\operatorname{gcd}\left(c^{m}+1, c^{n}-1\right)$ either is 1,2 or $c^{d}+1$, in which case $n / d$ is even.

As $\frac{s}{t}+1>2$, with $\frac{\alpha(a)}{f(a)}=p^{v}$ and $\frac{s}{t}=p^{u}$ we get that $u=\operatorname{gcd}(v, u)$ and $v / u$ is even. So

$$
\frac{\alpha(a)}{f(a)}=\left(\frac{s}{t}\right)^{o(a)}
$$

with $o(a)$ even.

The case $\alpha(a) \leq f(a)$ is obtained by reversing the roles of $\alpha(a)$ and $f(a)$. 


\section{EGQS OF ORDER $\left(t^{2}, t\right)$ WITH (*) ARE STGQs}

In this section, we prove the surprising fact that if $\left(\mathcal{S}^{x}, H\right)$ is an EGQ with parameters $\left(t^{2}, t\right)$ such that (*) is satisfied for $H$, then it is an STGQ. We use the fact that $t$ is a prime power (consult the monograph [47], or see the Chen-Hachenberger result).

We first make some considerations under the assumption that only the members of $\mathcal{F}$ are abelian $\left(\left(\mathcal{F}, \mathcal{F}^{*}\right)\right.$ being the associated Kantor family).

Let $a$ be an element of $H^{\times}$with only fixed points of the line $L_{a} \mathrm{I} x$. By $\left(^{*}\right)$, the number of such points is $s+1=t^{2}+1$. Let $U$ be any line not concurrent with $L_{a}$; then the triad $\left\{L_{a}, U, U^{a}\right\}$ has precisely $t+1$ centers, and all these centers are obviously fixed by $a$. Now let $X$ and $Y$ be arbitrary nonconcurrent lines in $K(a)$, the set of fixed affine lines of $a$. Let $V$ be a line not incident with $x$ which meets both $X$ and $Y$, and let $\mu \in H_{V}$ map $X$ to $Y$. Then $[a, \mu]=\mathrm{id}$, as $[a, \mu]$ fixes $L_{a}$ and $\operatorname{proj}_{x} V$ pointwise, while $V$ is fixed (noting that $X$ and $Y$ are centers of the line triad $\left\{L_{a}, V, V^{a}\right\}$ ). So $\mu \in C_{H}(a)$, and from this observation it easily follows that $C_{H}(a)$ acts transitively on $K(a)$, readily implying that $f(a)=|K(a)|$ is prime power. So the Transfer Formula (Theorem 5.9p for $a$ can be applied:

$$
\frac{s}{f(a)}=\frac{\alpha(a)}{f(a)}=\left(\frac{s}{t}\right)^{o(a)}=t^{o(a)}
$$

with $o(a) \in 2 \mathbb{Z}$. If $f(a) \leq t^{2}=s$, then $o(a) \geq 0$. As $f(a)>1, o(a)=0$ and so $\alpha(a)=s=$ $f(a)$. If $f(a)>s$, then $f(a) \geq t^{4}$, contradiction.

Remark 6.1. We have the same arithmetic information at hand regarding the number of fixed lines of line stabilizers as for $(*)$-STGQs of order $\left(t^{2}, t\right)$. It might be possible to pass from this point to the conclusion of Theorem 6.2 below without assuming $(*)$ for the entire elation group.

If we now assume that the entire elation group has $(*)$, a short proof of the following is possible.

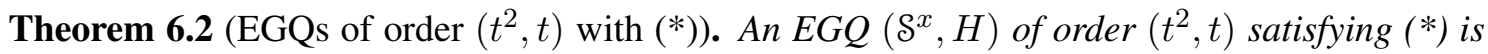
an $S T G Q$. In particular, $x$ is a regular point.

Proof. Let $b \sim x \neq b, c \sim x \neq c, b \neq c$, and consider $H_{b, c}$. Suppose that some element $\nu$ of $H_{b, c}$ is not a symmetry; then there is an affine line $U$ such that $U \nsim U^{\nu}$. But then any of the $t+1$ centers of $\left\{B, U, U^{\nu}\right\}$ is fixed by $\nu$, forcing $\nu$ to be the identity, as $x b$ and $x c$ are pointwise fixed (cf. Theorem 2.4). So $H_{b, c}$ only consists of symmetries, and $\left(\mathcal{S}^{x}, H\right)$ is an STGQ.

Theorem 6.2 naturally generalizes to the infinite case, but such considerations will be done in a later paper. 


\section{PART II}

\section{EXISTENCE OF ROOT-ELATIONS}

\section{RoOT-ELATIONS}

In this section we will show that (many) pseudo root-elations exist for certain STGQs. In the entire section, we will be mainly concerned with STGQs satisfying (*). Some intermediate lemmas will be stated in such a way that they will also be valid in the non-(*) case, which will be handled near the end of the paper. At several stages, we will obtain results in multiple ways, each adding additional insight in the structure of the elation group, $H$ (and $\mathcal{S}^{x}$ ). The different approaches often generalize to different more global properties. We will also obtain information about the exponents of skew elation groups as a corollary of the obtained results.

7.1. Existence of root-elations, I. In this subsection we suppose $(*)$ for the STGQ $\left(\mathcal{S}^{x}, H\right)$. In a first instance, we let $s \neq t$ if $s$ is even, although we will consider the case of even $s=t$ in another paper of the series.

Let $L$ be an arbitrary affine line, and put $[L]=\operatorname{proj}_{x} L$; let $l \mathrm{I} L \mathrm{I} l \neq x$, and let $[M] \neq[L]$ be arbitrary on $x$. Put $M=\operatorname{proj}_{l}[M]$. Let $\alpha$ be any element of $H_{L}^{\times}$; then (*) implies $H_{L} \leq C_{H}(\alpha)$. By Corollary 5.8, $\alpha$ fixes precisely $s$ affine lines (which are in $[L]^{\perp}$ ). Let $\mathcal{B}_{\alpha}$ be the set of these lines, and let $\mathcal{P}_{\alpha}$ be the set of $s^{2}$ points "in" $\mathcal{B}_{\alpha}$. Note that for any $m, m^{\prime} \mathrm{I}[M] \mathrm{I} m, m^{\prime} \neq x$, we have $H_{m}=H_{m^{\prime}}$. The following is obvious, and will be used without further notice (it is only valid because $\mathcal{S}^{x}$ satisfies (*)! - it is also true when $s=t$ is even; in that case, $\mathcal{B}_{\alpha}$ could be a larger set of lines, though):

Lemma 7.1. $\gamma \in H$ centralizes $\alpha$ if and only if $\gamma$ sends at least one point of $\mathcal{P}_{\alpha}$ to some other point of $\mathcal{P}_{\alpha}$. If this is the case, it stabilizes $\mathcal{P}_{\alpha}$ (and $\mathcal{B}_{\alpha}$ ) globally.

It is easy to see that $C_{H}(\alpha)$ "splits over $H_{L}$ and $H_{m}$ " - that is, $C_{H}(\alpha)=\left(C_{H}(\alpha) \cap H_{L}\right)\left(C_{H}(\alpha) \cap\right.$ $\left.H_{m}\right)=H_{L} C_{H_{m}}(\alpha)$ (for instance by using Lemma 7.1, and using the respective orders of the groups under the consideration in the last equality, cf. Corollary 5.8). Let $[\alpha]$ be the subset of $[L]$ of points incident with a line of $\mathcal{B}_{\alpha}$.

Lemma 7.2. $C_{H_{m}}(\alpha)$ acts transitively on $\mathcal{B}_{\alpha}$, so also on $[\alpha]$. Whence each point of $[\alpha]$ is incident with a constant number of affine lines fixed by $\alpha$. This number is $s /|[\alpha]|=: r>0$, and also equals $\left|C_{\mathbb{S}}(\alpha)\right|$.

Remark 7.3. The first two statements of Lemma 7.2 are also valid for the $s=t$ even case.

We will show that ${ }^{*}$ ) forces $C_{H_{m}}(\alpha)$ to split over $\mathbb{S}$ and $H_{M}$. To do this, put $U=M^{\alpha^{-1}}$, and let $k=\left|H_{U} \cap H_{M}\right|=\left|\{[L], M, U\}^{\perp}\right|$. Note that $H_{U} \cap H_{M}$ is precisely $C_{H_{M}}(\alpha)$. Since $H_{M}$ is abelian, $H_{U} \cap H_{M}$ fixes the $s / k$ affine lines on $\operatorname{proj}_{[M]} l^{\alpha^{-1}}$ which also meet $\{[L], M\}^{\perp}$. By symmetry, $H_{U} \cap H_{M}$ also fixes (at least) $s / k$ affine lines on $\operatorname{proj}_{[M]} l$. By way of contradiction, suppose that $k<|[\alpha]|$. Then $k<s / r$. So each element of $C_{H_{M}}(\alpha)$ fixes strictly more affine lines through $\operatorname{proj}_{[M]} l$ than $\alpha$ does through $L \cap[L]$ - call this property (L).

Now suppose that the value $r=r(\alpha)$ is maximal for the function

$$
r: \cup_{Y \in \mathcal{F}} Y^{\times} \longmapsto\{1, \ldots, t\}
$$

which sends each $\eta \in \cup_{Y \in \mathcal{F}} Y^{\times}$to the value $\frac{s}{|[\eta]|}$; then by $(\mathrm{L})$, we have that $k=s / r(\alpha)=|[\alpha]|$. Whence 


$$
C_{H_{m}}(\alpha)=\left(C_{H_{m}}(\alpha) \cap \mathbb{S}\right)\left(C_{H_{m}}(\alpha) \cap H_{M}\right)=C_{\mathbb{S}}(\alpha) C_{H_{M}}(\alpha) .
$$

Note that $s / r$ (and so $\left|C_{H_{M}}(\alpha)\right|$ ) is independent of the choice of $M$ (and the choice of $l \mathrm{I} L$ ). This implies that for any affine line $V I l$, we have that $\left|\left\{[L], V, V^{\alpha^{-1}}\right\}^{\perp}\right|=s / r$, in other words, $V$ contains precisely $s / r$ points of $\mathcal{P}_{\alpha}$. An easy counting argument leads to the fact that $r(\alpha)=t$. Let $\beta \in C_{H_{M}}(\alpha)=H_{M} \cap H_{U}$; as $r(\beta)$ (obvious notation) $\geq s / k=r(\alpha)=t$, it follows that $r(\beta)=t$.

Proposition 7.4 (Transfer). Let $\nu \in A^{\times}, A \in \mathcal{F}$. If $r(\nu)=t$ and $[\nu, \mu]=\mathrm{id}$, with $\mu \in B^{\times}$and $B \in \mathcal{F} \backslash\{A\}$, then $r(\mu)=t$.

Now suppose $\gamma \in \cup_{Y \in \mathcal{F}} Y^{\times}$is such that $r(\gamma)$ is the second largest value for $r(\cdot)$, and suppose furthermore that $r(\gamma)<t$. Suppose that $\gamma \in C \in \mathcal{F}$. Let $\omega \in D^{\times}, D \in \mathcal{F} \backslash\{C\}$, be such that $[\gamma, \omega]=$ id. If $r(\omega)>r(\gamma)$, then $r(\omega)=t$ by assumption, and then we have seen that $r(\gamma)=t$ by Transfer, contradiction. So $r(\omega) \leq r(\gamma)$ for all such $\omega$, implying equality for all such $\omega$. But then we have seen that this forces $r(\gamma)=t$, contradiction.

The following structural result follows.

Theorem 7.5 (Centrality $\left.\equiv(\text { MSTGQ1) })^{b}\right)$. Let $\left(\mathcal{S}^{x}, H\right)$ be an STGQ of order $(s, t)$, where $s$ is odd if $s=t$. Suppose $(*)$.

(i) For any affine line $L, H_{L}=H_{\left[\operatorname{proj}_{L} x\right]}$, that is, each element of $H_{L}$ fixes $\operatorname{proj}_{L} x$ linewise.

(ii) For each element $\alpha$ of $H_{L}^{\times},|[\alpha]|=s / t$; in particular, if $s=t$ is odd, the affine fixed line structure of $\alpha$ precisely consists of the tines through $\operatorname{proj}_{L} x$.

(iii) $Z(H)=\mathbb{S}$.

7.2. Exponents. In this subsection, we suppose that if $s \neq t,(*)$ holds, and that the elements of $\mathcal{F}$ are elementary abelian. Put $p=\operatorname{char}\left(\mathcal{S}^{x}\right)$.

Lemma 7.6. Let $G$ be a nilpotent group of class 2 , and let $a, b, c \in G$.

(i) For any natural number $m$, we have that $\left[a^{k}, b\right]=[a, b]^{k}$.

(ii) For any natural number $m$, we have that

$$
(a c b)^{m}=a^{m} c^{m} b^{m}[b, a]^{m(m-1) / 2}[c, a]^{m(m-1) / 2}[b, c]^{m(m-1) / 2} .
$$

Proof. The first part is easy. The second part follows by (i) and induction.

Take any element $s$ of $\mathbb{S}^{\times}$, and consider any point $y \sim x \neq y$. By Theorem 7.5 (i), there exist $a \in H_{[y]}^{\times}$. Choose $a$ to have order $p$, and note that $[s, a]=\mathrm{id}$. Under the standing hypothesis of the present subsection, we have that $s a$ fixes some affine line, so

$$
\mathrm{id}=(s a)^{p}=a^{p} s^{p}=s^{p},
$$

so $\mathbb{S}$ has exponent $p$. So by Theorem 7.5 (iii), it follows that $\mathbb{S}$ is elementary abelian.

Proposition 7.7. Let $\left(\mathcal{S}^{x}, H\right)$ be an $S T G Q$ of order $(s, t)$. Let $\left(\mathcal{F}, \mathcal{F}^{*}\right)$ be the associated Kantor family. Suppose that the elements of $\mathcal{F}$ are elementary abelian, and that either $s=t$ is odd, or $(*)$ holds if $s \neq t$. Then the following properties hold.

(i) The elements of $\mathcal{F}^{*}$ have order $p$. In particular, the exponent of $\mathbb{S}$ is $p$.

(ii) If st is odd, $H$ has exponent $p$. If st is even, it has exponent 4. 
Proof. Part (i) has already been obtained, so we only deal with part (ii). As $H / \mathbb{S}$ is abelian and generated by elementary abelian groups (namely the groups $A \mathbb{S} / \mathbb{S}$ with $A \in \mathcal{F},\left(\mathcal{F}, \mathcal{F}^{*}\right)$ the associated Kantor family), it is also elementary abelian. (Note that this already implies that for any $\mu \in H, \mu^{p^{2}}=\mathrm{id}$, so the exponent of $H$ is either $p$ or $p^{2}$.) Let $A, B \neq A$ be in $\mathcal{F}$; then $H=A B \mathbb{S}$. Let $\nu$ be arbitrary in $H$, so that we can write $\nu=a b s$ for $a \in A, b \in B, s \in \mathbb{S}$. As $[H, H] \leq \mathbb{S} \leq Z(H)$ by the assumptions and the previous subsection, $H$ has nilpotence class 2 . So by Lemma 7.6 .

$$
\nu^{p}=(a b s)^{p}=a^{p} b^{p}[b, a]^{p(p-1) / 2}=[b, a]^{p(p-1) / 2} .
$$

If $p$ is odd, the latter term is id, so then $H$ has exponent $p$.

The case $s=t$ even will be handled in a separate paper, and needs a different, indirect, approach. It is much harder.

7.3. Existence of root-elations, II. In this subsection we suppose that for the STGQ $\mathcal{S}^{x}$ with Kantor family $\left(\mathcal{F}, \mathcal{F}^{*}\right),(*)$ holds. Moreover, we suppose that the elements of $\mathcal{F}$ are elementary abelian. If $s=t$ (when $(*)$ is automatically satisfied), we furthermore suppose that $s$ is odd - as in most parts of this section, we will handle the even square case separately. The goal is to obtain nontrivial root-elations in an entire different manner as before. (The results will be slightly weaker at the level of root-elations.) To that end, we will obtain some group theoretical results which have independent interest.

Before proceeding, we first recall the following result by Alperin and Glauberman, of [1].

Theorem 7.8 ([1]). If $A$ is an abelian subgroup in a (finite) $p$-group $P$ of exponent $p$ and class at most $p+1$, then there is a normal abelian subgroup $B$ of the same order contained in the normal closure of $A$ in $P$.

Case $s>t$. First suppose $s \neq t$, and let $A=H_{L}, L$ an affine line. By assumption, $A$ is elementary abelian, so that Theorem 7.8 and $\$ 7.2$ imply that $\left\langle A^{H}\right\rangle$ contains an elementary abelian subgroup $\mathbb{A}$ of order $s>t$, which is normal in $H$. We note the following properties:

- $\mathbb{A}$ fixes $\operatorname{proj}_{x} L$ pointwise (by (*)).

- Since $|\mathbb{A}|>t$, the previous point implies $\mathbb{A}$ contains elements $\alpha$ fixing some affine line in $\left(\operatorname{proj}_{x} L\right)^{\perp}$, say $M$.

- It follows that $\left\langle\alpha^{H}\right\rangle \leq \mathbb{A}$.

Let $\operatorname{proj}_{M} x=m$. Let $\eta$ be any nontrivial symmetry, and put $M^{\eta}=M^{\prime}$. Then $\alpha \eta$ is an element of $H_{m} \backslash \mathbb{S}$ which sends $M$ to $M^{\prime}$. As $\alpha \eta$ fixes $x m$ pointwise, it must fix some affine line $M^{\prime \prime}$ meeting $x m$, so that clearly there must be an $h \in H$ such that

$$
\alpha^{h}=\alpha \eta \text {. }
$$

(Note that $\alpha$ and $\alpha \eta$ have the same action on the lines incident with $x$.) So $[\alpha, h]=\eta \in \mathbb{A}$. We have shown

Lemma 7.9. $\mathbb{S} \leq \mathbb{A}$. As a corollary, $\mathbb{S}$ is elementary abelian.

Theorem 7.10 (Existence of root-elations (for $s \neq t,(*))$ ). Let $\left(\mathcal{S}^{x}, H\right)$ be an STGQ of order $(s, t)$ for which $\left(^{*}\right)$ holds, and such that $s \neq t$ if $s$ is even. Suppose furthermore that affine line stabilizers are elementary abelian. Then for any $L$ not incident with $x, H_{L}$ contains at least $s / t>1$ elements which fix $\operatorname{proj}_{L} x$ linewise, that is, $H_{L}$ contains at least $s / t$ root-elations with i-root $\left(x, x \operatorname{proj}_{L} x, \operatorname{proj}_{L} x\right)$.

Proof. Put $\operatorname{proj}_{L} x=l$. Since $\mathbb{A}$ is a subgroup of $H_{l}$ and contains $\mathbb{S},\left|\mathbb{A}_{L}\right|=s / t$. The proof follows from the fact that $\mathbb{A}$ is abelian, so that $\left[\mathbb{A}_{L}, \mathbb{S}\right]=\{\mathrm{id}\}$. 
Square STGQs. Now suppose that $s=t$ is odd, and let $L$ be an affine line. Consider the nontrivial group $Z(H) \leq \mathbb{S}$; then $\left\langle Z(H), H_{L}\right\rangle$ is an elementary abelian group of order $s r>s$, with $r=|Z(H)|$. Now carry out the same argument as for the case $s \neq t$ with $A=H_{L}$, to conclude that

- $\mathbb{A}$ is an elementary abelian normal subgroup of $H$ of order $s r>s$;

- $\mathbb{S} \leq A$, so that $\mathbb{S}$ is abelian.

Theorem 7.11 (Existence of root-elations (for $s=t$ odd)). Let $\left(\mathcal{S}^{x}, H\right)$ be an STGQ of order $s$ with $s$ odd. For any $L$ not incident with $x, H_{L}$ contains at least $|Z(H)|>1$ elements which fix $\operatorname{proj}_{L} x$ linewise, that is, $H_{L}$ contains at least $|Z(H)|$ root-elations with $i$-root $\left(x, x \operatorname{proj}_{L} x, \operatorname{proj}_{L} x\right)$.

To eventually complete the square GQs, we need to consider the case $s=t$ even, in which we cannot employ the property that elements of $\cup_{A \in \mathcal{F}} A^{*} \backslash \mathbb{S}$ necessarily fix affine lines.

7.4. Abelian subgroups of metabelian groups. Recall that a group $G$ is called metabelian if its first derived group is abelian. So $G$ is metabelian if and only if there is a normal abelian subgroup $A$ such that $G / A$ also is abelian.

Theorem 7.12 (J. D. Gillam [15]). Let $G$ be a finite metabelian group, and let $B$ be an abelian subgroup of $G$ of maximal size. Then $G$ contains a normal abelian subgroup $C \leq\left\langle B^{G}\right\rangle$ for which $|B|=|C|$.

Following the (same) proof of A. Mann [21], one obtains the following variation on Theorem 7.12

Theorem 7.13. Let $G$ be a finite metabelian group, and suppose $N$ is a normal subgroup of $G$. Let $B$ be an abelian subgroup of $N$ of maximal size (in $N$ ). Then $G$ contains a normal abelian subgroup $C \leq\left\langle B^{G}\right\rangle \leq N$ for which $|B|=|C|$.

Proof. (Sketch.) Let $G$ and $N$ be as in the statement of the theorem. Choose $B$ among the abelian subgroups in $\left\langle B^{G}\right\rangle \leq N$ with size $|B|$ in such a way that it has maximal intersection with $[G, G]$. Assume that it is not normal in $G$ (by way of contradiction). Then $N_{G}(B) \neq G$, so the normalizer condition implies that we can choose an element $h$ in $N_{G}\left(N_{G}(B)\right) \backslash N_{G}(B)$. Define $A=B B^{h}$, and note the following properties:

- $B^{h} \neq B$ so that $A \neq B$;

- $A \leq N$;

- $B$ and $B^{h}$ normalize each other, so $A=B[B, h]$ has class 2 and $Z(A)=B \cap B^{h}(B$ is maximal abelian in $A)$.

Define $E=Z(A)(B \cap[G, G])[B, h]$; then $E \leq\left\langle B^{G}\right\rangle \leq N$, and $|B| \leq|E|$ (note that $A=B E$, and $B \cap B^{h} \leq E \cap B$ ). Since $E$ is abelian, equality holds. But $E \cap[G, G]>B \cap[G, G]$ (as $[B, h] \notin B)$, contradiction.

Now let $\left(\mathcal{S}^{x}, H\right)$ be an STGQ with $\left(^{*}\right)$, and metabelian $H$. Note that when $\left(^{*}\right)$ is satisfied, $H$ is metabelian if and only if $\mathbb{S}$ is abelian. We have proved that this is already the case if $s$ is even and $s \neq t$ (assuming that affine line stabilizers are elementary abelian).

Choose any affine line $L$; let $u$ be such that $L I u \sim x \neq u$. Then $H_{u}$ is normal in $H$. Let $B$ be abelian of maximal size in $H_{u}$; then $|B|>s$ since $H_{L}$ is abelian and $Z(H) \leq \mathbb{S} \leq H_{u}$, while $\mathbb{S} \cap H_{L}=\{\mathrm{id}\}$. So by Theorem 7.13, $B$ can be taken to be normal in $H$. By (*), each element $b$ of $B \backslash \mathbb{S}$ fixes some affine line in $x u^{\perp}$, and so $[b, H]=\mathbb{S} \leq B$. On the other hand, $[b, \mathbb{S}]=\{\operatorname{id}\}$ implies that $b$ is a root-elation (with i-root $(u, x u, x)$ ). The following theorem follows immediately:

Theorem 7.14. Suppose $\left(\mathcal{S}^{x}, H\right)$ satisfies $\left(^{*}\right)$, and let $\mathbb{S}$ be abelian. Then for each point $y \sim x \neq y$, $\left|H_{[y]}\right|>s / t$. In particular, if $s$ is even with $s \neq t$ and affine line stabilizers are elementary abelian, we have this conclusion. 
Remark 7.15 (Metabelian $H$ in even characteristic). Suppose that $\mathbb{S}$ is an elementary abelian 2group, and let $|\mathbb{S}|=2^{n}=t$. We see $\mathbb{S}$ as an affine $n$-space $\Pi$ over $\mathbb{F}_{2}$. Then we have a natural homomorphism

$$
\eta: H \mapsto \mathbf{A G L}_{n}(2)
$$

defined by conjugation; its kernel is $C_{H}(\mathbb{S})$. It might be interesting to study the action of the involutions in $H$ on the space $\mathbb{S}$.

7.5. STGQs of order $\left(t^{2}, t\right)$. For STGQs of order $\left(t^{2}, t\right)$ with $(*)$, there is a general combinatorial approach, independent of the characteristic, and inspired by the beginning of this section, which directly leads to centrality of $\mathbb{S}$.

Theorem 7.16. An STGQ of order $\left(t^{2}, t\right)$ with $(*)$ is central.

Proof. Let $L \mp x$, and let $U \mathrm{I} x$ be arbitrary but different from $\operatorname{proj}_{x} L$. Put $Y=\{U, L\}^{\perp} \backslash$ $\left\{\operatorname{proj}_{x} L\right\}$. Let $r$ be any point of $\operatorname{proj}_{x} L$ different from $x$ and not incident with $x$. Let $R$ be any line on $r$ not on $x$; then $R$ meets $Y$ in $t$ different points (as the order of $\mathcal{S}^{x}$ is $\left(t^{2}, t\right)$ ). Clearly, $H_{L}$ acts transitively on the lines incident with $r$ and different from $\operatorname{proj}_{x} L$. So if $\beta \in H_{L}$ fixes $R$, the fact that $H_{L}$ is abelian implies that $\beta$ fixes $r$ linewise. In particular, $H_{L} \cap H_{R}$ yields a group of $\left(r, \operatorname{proj}_{x} L, l\right)$-elations of size $t\left(l=x^{\perp} \cap L\right)$.

Now let $\alpha \in H_{L}^{\times}$be arbitrary. Let $V \in Y$ be arbitrary; then $\left\{\operatorname{proj}_{x} L, V, V^{\alpha}\right\}^{\perp}$ has size $t+1$, two of its elements being $U$ and $L$. Let $L^{\prime} \in\left\{\operatorname{proj}_{x} L, V, V^{\alpha}\right\}^{\perp} \backslash\{U, L\}$; then $\alpha \in H_{L^{\prime}} \cap H_{L}$, so that the first part of the theorem yields that $\alpha$ fixes $l$ linewise. 


\section{PART III}

\section{SQUARE ODD STGQS - CLASSIFICATION}

\section{SQUARE STGQS OF ODD ORDER - CLASSIFICATION}

We are ready to formulate and prove our main result for odd square STGQs. For the rest of this section, the GQ $\mathcal{S}^{x}$ will be a thick STGQ of odd order $s$. By $\$ 7.1, \mathcal{S}^{x}$ is central.

8.1. Structure of odd STGQs. We deduce some structural results from 87.1 . The following theorem is essentially contained in [12], and uses the Payne-derived quadrangle $\mathcal{P}\left(\mathcal{S}^{x}, x\right)$. We give a short different proof here using centrality.

Lemma 8.1. We have that (*) holds, so that $\mathbb{S}$ is the center of $H$.

Proof. Denote the group of symmetries about $x$ by $\mathbb{S}$ as usual. As $x$ is a regular point, there is a projective plane $\pi_{x}$ of order $q$ associated to $x$. Let $y \sim x \neq y$ be any point; then $H_{y}$ induces an automorphism group $H_{y} / \mathbb{S}$ on $\pi_{x}$ that clearly fixes all the points on $x y$ of the plane. So $H_{y}$ fixes any point of the line $x y$ of $\mathcal{S}$. In fact $H / \mathbb{S}$ induces a translation group of the translation plane $\pi_{x}$, so $H / \mathbb{S}$ is elementary abelian. By Theorem 5.1, (*) holds, so that by Theorem 7.5 (iii), $Z(H)=\mathbb{S}$.

We sum up some extra structural properties in the next theorem.

Theorem 8.2 (Structure theorem). Let $\mathcal{S}=\left(\mathcal{S}^{x}, H\right)$ be an $S T G Q$ of order $q$, $q$ an odd prime power, and let $\left(\mathcal{F}, \mathcal{F}^{*}\right)$ be the 4-gonal family arising from (w.r.t. any point not collinear with $x$ ). The following properties hold.

(i) The members of $\mathcal{F}^{*}$ are abelian.

(ii) $\mathcal{S}^{x}$ satisfies $(M)_{x}$.

(iii) $H$ is a special group of exponent $p$, $q$ being a power of the prime $p$.

Proof. (i) Follows from Theorem 5.1 and Lemma 8.1 .

(ii) Let $M \pm x$ be arbitrary, and let $m$ be so that $x \sim m \mathrm{I} M$. Then $H_{m}$ is abelian, and so $H_{M}$ is a normal subgroup of $H_{m}$. Since $\mathbb{S} \leq H_{m}$, it follows that $H_{M}$ fixes every line incident with $m$. So (ii) is satisfied.

(iii) By Proposition 7.7, $H$ has exponent $p$. As $[H, H] H^{p}=\Phi(H)$, it follows that $\Phi(H)=$ $[H, H]$. Now consider any two elements $\alpha, \beta \in H$; then $[\alpha, \beta]$ is a symmetry about $x$ (look at the action on $\pi_{x}$ ), so $[H, H] \leq Z(H)=\mathbb{S}$ (for the latter equality, confer Theorem 7.5). Let $A, B \in \mathcal{F}$ be distinct, and choose $\alpha \in A^{\times}, \beta, \beta^{\prime} \in B^{\times}$; then $[\alpha, \beta]=\left[\alpha, \beta^{\prime}\right]$ if and only if $\left[\beta^{\prime} \beta^{-1}, \alpha\right]=$ id if and only if $\beta^{\prime} \beta^{-1}$ is a symmetry in $Z(H)$, cf. Corollary 5.8. This implies that $\beta=\beta^{\prime}$, and one deduces that $|[H, H]| \geq q$, so $[H, H]=Z(H)$.

Lemma 8.3 (Projection Lemma). No proper subgroup of H projects onto $T$.

Proof. By the previous lemma, we know that $\Phi(H)=\mathbb{S}$. Now, by way of contradiction, let $R \leq H \neq R$ project on $T$, and suppose $M \leq H$ is a proper maximal subgroup containing $R$. Then $M$ contains $\Phi(H)$, so also $\mathbb{S}$; this leads to the fact that $M=H$, a contradiction. 
8.2. Property (AR1). Take $l \in A \in \mathcal{F}$; then by Corollary 5.8

$$
[l, H]=\left[l, A^{*} B\right]=\left[l, C_{H}(l) B\right]=[l, B]=[H, H]=\mathbb{S} .
$$

This implies the following property which generalizes antiregularity:

(AR1) No triad $\{U, V, W\}$ of lines, with $U \mathrm{I} x$ and center $X \mathrm{I} x$, has more than one center which is not incident with $x$.

In the next section we will further exploit (AR1) to show that EGQs with (AR1) are classical.

8.3. Main result. A group $G$ is of semifield type if there is some prime power $q$ for which $|H|=$ $q^{3}$, and such that there are normal elementary abelian subgroups $M$ and $N$ of order $q^{2}$ for which

(i) $H=M N$;

(ii) $[m, n]=$ id implies $m \in M \cap N$ or $n \in M \cap N$ for all $m \in M$ and $n \in N$.

In [18], the author shows that from a semifield group $G$ and a pair $(M, N)$ such as above, a semifield $\mathbb{S}(M, N)$ can be constucted of order $q$, and conversely, any semifield $\mathbb{S}$ gives rise to a semifield group in a natural way (if the plane is not Desarguesian, the group is simply the collineation group generated by all elations). In fact, the corresponding semfield group acts naturally on the semifield plane $\Pi(\mathbb{S})$ which is coordinatized by $\mathbb{S}$.

Let $A^{*}, B^{*}$ be distinct elements of $\mathcal{F}^{*}$; then $A^{*}$ and $B^{*}$ are elementary abelian and normal (since they both contain $[H, H]$ ), and (ii) is obviously satisfied by Corollary 5.8. So $H$ is a group semifield type with respect to any two distinct members of $\mathcal{F}^{*}$. We now address the following result of Hiramine:

Theorem 8.4 (Y. Hiramine [18]). Let $G$ be a group of semifield type of order $q^{3}$, with $q=p^{h}, p a$ prime and $h \in \mathbb{N}^{\times}$. Let $W_{G}=W$ be the set of all abelian subgroups of order $q^{2}$.

- If $p=2$, then $|W|=2$.

- If $p>2$, then $|W|=p^{r}+1$ for some natural number $r \leq h$, and $H \curvearrowright W$ contains the natural (2-transitive) action of $\mathbf{P S L}_{2}\left(p^{r}\right)$ of degree $p^{r}+1$.

Also, $|W|=q+1$ if and only if the corresponding plane is Desarguesian; in that case, $G$ is isomorphic to the following group:

$$
\left\{\left(\begin{array}{ccc}
1 & \alpha & c \\
0 & \mathbb{I} & \beta^{T} \\
0 & 0 & 1
\end{array}\right) \mid \alpha, \beta \in \mathbb{F}_{q^{2}}, c \in \mathbb{F}_{q}\right\} \cong \mathcal{H}_{1}(q),
$$

with standard matrix multiplication, and where $\mathbb{I}$ is the identity $2 \times 2$-matrix.

As $\left|\mathcal{F}^{*}\right|=q+1$, we have obtained the following theorem.

Theorem 8.5. $H$ is isomorphic to the classical semifield group $\mathcal{H}_{1}(q)$.

Now consider any line $[A] \mathrm{I} x, A \in \mathcal{F}$, and consider $A^{H}$. By (AR1) (see also Corollary 5.8), any two of the $q$ members of $A^{H}$ meet only in the identity; it follows that $A^{H} \cup \mathbb{S}$ is a partition of $A^{*}$ into $q+1$ subgroups of order $q$. Whence there arises a translation plane $\Pi_{A}$ of order $q$ for which $A^{*}$ is the translation group. Moreover, $H$ acts faithfully as a collineation group on $\Pi_{A}$, and since for any $B \in \mathcal{F} \backslash\{A\}, B$ fixes the point at infinity of $\Pi_{A}$ corresponding to $\mathbb{S}$ and acts sharply transitively on the other points at infinity, $\Pi_{A}$ is a semifield plane. By Theorem $8.4, \Pi_{A}$ is Desarguesian. 
Remark 8.6. Note that the planes $\Pi_{A}$ are nothing else than the planes we construct geometrically in the next section by using (AR1).

Consider the projective plane $\Pi_{x}$; it is the projective completion of the translation plane which arises from the partition in the group $H / \mathbb{S}$ defined by $\left\{A^{*} / \mathbb{S} \mid A^{*} \in \mathcal{F}^{*}\right\}$. As $H \cong \mathcal{H}_{1}(q)$ and as the number of maximal elementary abelian subgroups of $\mathcal{H}_{1}(q)$ is $q+1, \Pi_{x}$ must be Desarguesian. (Note that $[.,]:. H / \mathbb{S} \times H / \mathbb{S} \mapsto \mathbb{S}$ defines an alternating form over $\mathbb{F}_{q}$.)

Theorem 8.7. $\mathcal{S}^{x} \cong \mathcal{W}(q)$ and $H \curvearrowright \mathcal{S}^{x}$ is permutation equivalent with $\mathcal{H}_{1}(q) \curvearrowright \mathcal{W}(q)^{z}$ for any point $z \in \mathcal{W}(q)$, the latter action being that as an elation group.

Proof. At this point, there are different ways to proceed.

[First method] One way is to construct explicitly an automorphism of $H$ which sends the Kantor family of $\mathcal{S}^{x}$ to the classical Kantor family of $\mathcal{W}(t)$. As this is done in the recent paper [14], we will not do this, and instead refer to loc. cit. — see also 8.5 below.

[Second method] Another way is to "extend (AR1)" to the property that all lines incident with $x$ are antiregular (again, we won't do it explicitly due to Ghinelli's paper). The result then follows from the fact that each plane $\Pi_{A}$ is Desarguesian and [27, 5.2.7].

[Third method] Observe that (AR1) is the geometrical formulation of te property "of symplectic type" — see 8.5 - and use Ghinelli's Theorem 8.14

Remark 8.8. - I proved Theorem 8.7 in 2009, and already announced it (a.o.) at the 2010 conference "Combinatorics 2010" in Verbania. It was also mentioned (without proof) in the proceedings paper of my talk [46], which was published in 2011. I explained several proofs of this result in a lecture at the "Buildings 2012" conference in Münster.

- The fact that $\mathcal{S}^{x} \cong \mathcal{W}(q)$ was also published in the paper [2] in 2015 .

8.4. Implications. The next set of theorems follows immediately.

Theorem 8.9. If $\left(\mathcal{S}^{x}, H\right)$ is an EGQ of order $q$ with $H \cong \mathcal{H}_{1}(q)$, then $\mathcal{S}^{x} \cong \mathcal{W}(q)$.

Theorem 8.10. If an EGQ of order $q$ admits an elation group of semifield type, we have the conclusion of Theorem 8.7

Theorem 8.11. An EGQ with at least one antiregular line is isomorphic to $\mathcal{W}(q)$.

Theorem 8.12. If $\left(\mathcal{S}^{x}, H\right)$ is an EGQ of odd order $q$ and $|Z(H)| \geq q$, then we have the conclusion of Theorem 8.7

Theorem 8.13. If $\left(\mathcal{S}^{x}, H\right)$ is an EGQ of order $q$ with only abelian components (in the corresponding Kantor family), then we have the conclusion of Theorem 8.7 
8.5. Appendix — Ghinelli's result. In [14], Ghinelli studies so-called "AS-configurations" in finite groups. Such a configuration is a set of subgroups $A_{0}, \ldots, A_{n+1}$ in a group $A$ of order $n^{3}(n \in \mathbb{N}, n \geq 2)$, such that $A_{i} A_{j} \cap A_{k}=\{\operatorname{id}\}$ for two by two distinct $i, j, k$, while each $A_{i}$ has size $n$. Moreover, it is asked that $A_{0} \unlhd A$. It is straightforward to observe that putting $\mathcal{F}:=\left\{A_{1}, \ldots, A_{n+1}\right\}$ and $\mathcal{F}^{*}:=\left\{A_{0} A_{1}, \ldots, A_{0} A_{n+1}\right\}$, one obtains a Kantor family of type $(n, n)$ in $A$, that is, an EGQ $\Gamma$ of order $n$ with elation group $A$. But there is more: the group $A_{0}$ obviously is a group of symmetries with center the elation point $(\mu)-$ in other words, $\Gamma^{\mu}$ is an STGQ. Ghinelli then proves the following result (which we formulate in terms of STGQs, rather than in terms of AS-configurations).

Theorem 8.14 (Ghinelli [14]). A square $S T G Q\left(\Gamma^{\mu}, A\right)$ with odd order $n$ and of symplectic type is isomorphic to $\mathcal{W}(n)$.

Symplectic type means that each conjugacy class different from $\{\mathrm{id}\}$ has a representative in $\cup_{j=0}^{n+1} A_{j}^{\times}$. This is nothing else than the group theoretical formulation of (AR1), which always holds for square STGQs of odd order.

\section{COMBinAtorial LEMMA}

In this section, we observe that [26, 1.3.2] also holds for GQs that satisfy (AR1). We then derive some interesting consequences.

Let $Y$ and $X$ be concurrent lines in a GQ $\Gamma$ of order $s$, and suppose (AR1) is satisfied "with respect to $X$ and $Y$." Put $X \cap Y=z$, and define a rank 2 incidence structure $\Pi(X, Y)=(\mathcal{P}, \mathcal{B}, \mathrm{I})$ as follows.

- PoINTs are the elements of $X^{\perp}$ not through $z$.

- LINES are of two types:

- sets $\{Z, X\}^{\perp} \backslash\{Y\}$, with $Z \in Y^{\perp}, Z$ I $z$;

- the points of $X \backslash\{z\}$.

- INCIDENCE is the expected one.

Theorem 9.1. $\Pi(X, Y)$ is an affine plane of order s.

Proof. Let $y$ be any point incident with $Y$, and different from $z$. Clearly, if $V, W$ are such that $Y \neq V$ I $y \mathrm{I} W \neq Y$, then

$$
\{V, X\}^{\perp} \cap\{W, X\}^{\perp}=\{Y\} .(\#)
$$

So all lines in $\Pi(X, Y)$ determined by $y$ are parallel. Suppose $V, W \in Y^{\perp}, V \pm z \pm W$, and $V \nsim W$. Then $\{V, X\}^{\perp}$ and $\{W, X\}^{\perp}$ meet in at most one line of $X^{\perp}$ not incident with $x$. Using (\#) it follows that this is precisely one. The theorem follows.

The set $X \backslash\{z\}$ is a parallel class of lines. The other parallel classes correspond to points of $Y \backslash\{z\}$. Clearly, an element of $\operatorname{Aut}(\Gamma)_{Y, X}$ induces an element of $\operatorname{Aut}(\Pi(X, Y))$.

Below, if $Y \mathrm{I} z$ and the element of $\mathcal{F}^{*}$ corresponding to $Y$ has $(*)$, we also say that "(*) $)_{Y}$ is satisfied." And if $(M)_{x}$ is true locally at $Y$ for $G$, we say that " $(M)_{z, Y}$ is satisfied."

Theorem 9.2. Let $Y$ and $X$ be distinct concurrent lines in the $E G Q\left(\Gamma^{z}, G\right)$ of order $s, X I z I Y$, and suppose $(A R 1)$ is satisfied with respect to $X$ and $Y$. If $\left(\Gamma^{z}, G\right)$ satisfies $\left({ }^{*}\right)_{Y}$, it also satisfies $(M)_{z, Y}$. 
Proof. The subgroup $G_{U}, U$ any line in $Y^{\perp}$ not incident with $z$, induces a translation group (of order $s^{2}$ ) of $\Pi(X, Y)$. (Note that $\left({ }^{*}\right)_{Y}$ is used.) Whence $G_{U}$ fixes all lines on $\operatorname{proj}_{U} z$, i.e., $(M)_{z, Y}$ is satisfied.

In particular, if $\Gamma^{z}$ satisfies (AR1) for all line pairs on $z$, and $\left(^{*}\right)$ holds, it also satisfies $(M)_{z}$.

Corollary 9.3. Under the assumption of the previous theorem, we have that $G_{y}$ is elementary abelian for any $y \in Y \backslash\{z\}$.

Now suppose again that $\left(\Gamma^{z}, G\right)$ is an EGQ of order $s$, with (AR1) for all line pairs on $z$. Then

$$
G_{U}^{\times} \cap G_{U^{\prime}}^{\times}=\emptyset
$$

for lines $U, U^{\prime}$ with $U \cap U^{\prime} \nsim z$. (Here non-concurrent lines are allowed!) So the only elements of $G$ which do not fix some affine line, are contained in a set of size $s-1$. It immediately follows that $z$ is a center of symmetry, so that $\Gamma^{z}$ is an STGQ. (Whence (*) holds.) The next corollary now follows from our analysis in the square case.

Theorem 9.4. Let $\Gamma^{z}$ be an EGQ of order s. If one of the following properties hold, then $\Gamma^{z} \cong$ $\mathcal{W}(s), s$ is odd, and the elation group is isomorphic to $\mathcal{H}_{1}(q)$.

- Each pair of lines incident with z satisfies (AR1).

- Some line of $\Gamma^{z}$ is antiregular.

Remark 9.5 (Even case). Let $\Gamma^{z}$ be an EGQ, and suppose $X I z I Y$ are distinct regular lines. Then it is easy to prove (for instance by applying the methods of [41, Chapter 2]) that $X$ and $Y$ are axes of symmetry. This is already sufficient for $\Gamma^{z}$ to be a TGQ. When $s$ is even, $\Gamma^{z}$ is also an STGQ in this case. And of course, if some line not on $z$ is regular, $\Gamma^{z} \cong \mathbf{Q}(4, s)$.

Note the following property, which can be obtained "directly" from (AR1).

Theorem 9.6. Let $\left(\mathcal{S}^{x}, H\right)$ be an EGQ of order $s$, with $(A R 1)$. Then $H$ has exponent $p, H$ being a p-group.

Proof. By (AR1), $(M)_{x}$ is satisfied, and $H_{U}^{\times} \cap H_{V}^{\times}=\emptyset$ for $U \neq V$ nonconcurrent affine lines. So $\{\mathbb{S}\} \cup_{U \mp x}\left\{H_{U}\right\}$ defines an equal sized partition of $H$, implying that $H$ has exponent $p$.

QUESTION. Does (ARl) force the GQ to be square?

Corollary 9.7. If the EGQ $\left(\mathcal{S}^{x}, G\right)$ of order $(s, t)$ has $(A R 1)$ and $s=t$, then $t$ is odd and $G \curvearrowright \mathcal{S}^{x}$ is permutation equivalent with $\mathcal{H}_{1}(t) \curvearrowright \mathcal{W}(t)^{z}$, with $z$ any point of $\mathcal{W}(t)$. 


\section{PART IV}

\section{STGQS WITH IDEAL SUBGQS}

\section{TAME GROUPS AND TWISTING}

In this section we introduce the process of "twisting;" it was first described in [42]. To start, we need the definition of tame group, which, for now, we describe as follows.

10.1. Tame groups. Call an elation group of an STGQ $\mathcal{S}^{x}$ tame if it satisfies $(M)_{x}$. We conjecture that "most" STGQs admit a tame elation group. Still, it could happen that other elation groups arise, as indicated in the examples section, and in this and the next section we will classify the STGQs with this property in a very precise way. For this purpose, we introduce twisting [42] - a way to construct other elation groups ("twists") starting from data

(certain EGQ, involution fixing certain subGQ elementwise).

10.2. Twisting. For now, $\mathcal{S}^{x}=\mathcal{S}$ is an $E G Q$ of order $\left(q^{2}, q\right)$, q even, with elation group H. Also, $\mathcal{S}^{\prime}$ is a subGQ of order $\left(s^{\prime}, q\right), s^{\prime}>1$, which is fixed elementwise by a nontrivial collineation $\theta$ of $\mathcal{S}$. By [51], we then have that $\mathcal{S}^{\prime} \cong \mathcal{W}(q)$, and that $\theta$ is an involution.

Suppose $W$ is the group of all whorls about $x$, and let $S_{2}$ be a Sylow 2-subgroup of $W$ which contains $H$. Then $S_{2}$ clearly has size $2 q^{5}$. Put $H^{\prime}=\theta H$, so that $S_{2}=H \cup H^{\prime}$. As $\mathcal{S}^{\prime} \cong \mathcal{W}(q)$, and as each point of $\mathcal{W}(q)$ is regular, one observes that for each point $z \not x$, the pair $\{x, z\}$ is regular, so that $x$ is a regular point of $\mathcal{S}$. This implies that two distinct subGQs of order $q$ containing $p$ can only intersect in a very restricted manner: either they share the lines through $x$ and the points (of the subGQs) incident with these lines, or they intersect in the points and lines of a dual grid of order $(1, q)$. Let $\theta^{\prime}$ and $\theta^{\prime \prime}$ be two distinct nontrivial involutions in $S_{2}$ that respectively fix the subGQs $\mathcal{S}_{\theta^{\prime}}$ and $\mathcal{S}_{\theta^{\prime \prime}}$ (of order $q$ ) pointwise. Suppose that they intersect in a dual grid as above. Then there is a point $z \not x$ for which $\{x, z\}^{\perp \perp} \subseteq \mathcal{S}_{\theta^{\prime}} \cap \mathcal{S}_{\theta^{\prime \prime}}$. Since both $\theta^{\prime}$ and $\theta^{\prime \prime}$ fix $z$, we immediately have a contradiction since $\theta^{\prime} \neq \theta^{\prime \prime}$ and $\left|\left(S_{2}\right)_{z}\right|=2$.

So subGQs of order $q$ that are fixed pointwise by a nontrivial involution in $S_{2}$ mutually do not share points not collinear with $x$. This implies that if $\mathcal{S}_{1}$ and $\mathcal{S}_{2} \neq \mathcal{S}_{1}$ are two such subGQs, there is some line $M I p$ so that

$$
M^{\perp} \cap \mathcal{S}_{1}=M^{\perp} \cap \mathcal{S}_{2} .
$$

Also, it follows easily that the number of such subGQs is $q^{2}$, and that the associated involutions are mutually conjugate in $S_{2}$. Note also that all whorls of $S_{2}$ which are not elations about $x$ are contained in $H^{\prime}$. The group $S_{2}$ is non-cyclic; if it were cyclic, then $H$ would be abelian, implying in its turn that there are more lines through a point than points incident with a line (since $\mathcal{S}$ is then a TGQ, cf. Chapter 8 of [26]). As $S_{2}$ is non-cyclic, a result of P. Deligne [11] implies that $S_{2}$ has at least three subgroups of size $q^{5}$ (one of which is $H$ ). Suppose $H^{\prime \prime} \neq H$ is a subgroup of $S_{2}$ of order $q^{5}$. If $H^{\prime \prime}$ does not contain any of the $q^{2}$ involutions of above, then $H^{\prime \prime}$ is an elation group ("first case"). If $H^{\prime \prime}$ contains at least one such involution ("second case"), it contains all of them since they are mutually conjugate, and since $H^{\prime \prime}$ is a normal subgroup of $S_{2}$ (as a group of index 2). In that case, put $H_{1}=H^{\prime \prime} \cap H$, and $H_{2}=H^{\prime} \cap H^{\prime \prime}$. So $\left|H_{1}\right|=\left|H_{2}\right|=q^{5} / 2$. Then it is straightforward to see that

$$
H_{1} \cup \theta\left[H \backslash H_{1}\right]=H^{-}
$$


is an elation group of size $q^{5}$. As the first and second case are equivalent, we keep using the notation of the second case. We put $H_{4}=H \backslash H_{1}$ and $H_{3}=\theta H_{4}$.

Suppose $L I x$, and let $y \mathrm{I} L \mathrm{I} x \neq y$. Define $H(x, L, y):=H_{[\{x, L, y\}]}$.

Second Hypothesis. For all $L \mathrm{I} x$ and $y \mathrm{I} L \mathrm{I} x \neq y$, we have that $|H(x, L, y)|=q^{2}$. Also, $H^{2} \leq$ $Z(H)$, where $H^{2}=\left\{h^{2} \mid h \in H\right\}$ and $Z(H)$ is the center of $H$.

Remark 10.1. We leave it as an exercise to the reader to show that $x$ is a center of symmetry.

Since $|H(x, L, y)|=q^{2}$ for all $L$ and $y$ as above, and since these groups generate $H$, it is straightforward to show that $Z(H)$ is the group of symmetries about $x$. In fact, one observes that $Z(H)=Z\left(H^{-}\right)$. Let $H(x, L, y)$ be a root-group; then $H(x, L, y)^{2} \leq Z(H)$, so that $H(x, L, y)^{2}=\{\mathrm{id}\}$. So all such root-groups are elementary abelian. Now consider $\theta \phi \in H^{-}$, where $\phi \in H_{4}$ is a non-trivial root-elation in $H(x, M, z)$ with $z \in \mathcal{S}_{\theta}$ which does not fix $\mathcal{S}_{\theta}$ (it is an easy exercise that such a $\phi$ exists for suitable $z$ ). Then $(\theta \phi)^{2}=\left[\theta, \phi^{-1}\right]=[\theta, \phi]$ clearly cannot be the identity, while it fixes $z$ linewise. So $(\theta \phi)^{2} \notin Z\left(H^{-}\right)$, and hence $H \not H^{-}$. One can now conclude the next theorem.

Theorem 10.2 (Twisting, [42]). Let $\mathcal{S}=\left(\mathcal{S}^{x}, H\right)$ be an EGQ of order $\left(q^{2}, q\right)$, where $q$ is even, which contains a subGQ $\mathcal{S}^{\prime}$ of order $(s, q), s>1$, fixed pointwise by a nontrivial automorphism $\theta$ of $\mathcal{S}$. If $H^{2} \leq Z(H)$, and if for all $L \mathrm{I} x$ and $y \mathrm{I} L \mathrm{I} x \neq y$, we have that $|H(x, L, y)|=q^{2}$, then there is an automorphism group $H^{\prime}$ of $\mathcal{S}$ such that $H^{\prime} \neq H$ and $\left(\mathcal{S}^{x}, H^{\prime}\right)$ is an EGQ.

Corollary 10.3 ([42]). Let $\mathcal{S}=\left(\mathcal{S}^{x}, H\right)$ be an EGQ of order $\left(q^{2}, q\right)$, where $q$ is even, which contains a subGQ $\mathcal{S}^{\prime}$ of order $(s, q), s>1$, which is fixed pointwise by a nontrivial automorphism $\theta$ of $\mathcal{S}$. Let $z \nsim x$ and suppose $z \sim z_{i} \sim x$ for $i=0,1, \ldots$, q. If all groups $H\left(x, x z_{i}, z_{i}\right) \leq H$ are elementary abelian and have size $q^{2}$, then there is an automorphism group $H^{\prime}$ of $\mathcal{S}$ such that $H^{\prime} ¥ H$ and $\left(\mathcal{S}^{x}, H^{\prime}\right)$ is an $E G Q$.

\section{STGQS WITH MORE THAN ONE ELATION GROUP ARE CLASSICAL}

In this section it is our goal to give a complete answer to a question of Payne (asked at the 2004 Pingree Park conference "Finite Geometries, Groups and Computation"). As the classical STGQ $\mathcal{H}\left(3, t^{2}\right)$ is the only known finite quadrangle which allows different (even nonisomorphic) elation groups, Payne asked whether this property characterized the quadrangle. We will show that the anser is "yes."

The (proof of the) next structure theorem is partly a prototype for the general classification of STGQs of order $\left(t^{2}, t\right)$ (even though it turns out that $\operatorname{char}\left(\mathcal{S}^{x}\right)=2$ ).

Theorem 11.1. Let $\mathcal{S}^{x}$ be an $S T G Q$ of order $(s, t)$ such that $x$ is an elation point w.r.t. at least two distinct elation groups. Then

(i) $t=s^{2}$ is a power of 2 ;

(ii) $\mathcal{S}^{x}$ contains $\mathcal{W}(t)$-subGQs through $x$ which are fixed pointwise by an involution;

(iii) any elation group w.r.t. $x$ is either tame or twisted;

(iv) there is a unique tame elation group $\widehat{E}$; furthermore, this group has the property that for each $y \in x^{\perp} \backslash\{x\}$, the $i$-root $(x, x y, y)$ is Moufang with root group in $\widehat{E}$, the latter being generated by all root-groups of the aforementioned type;

(v) any twisted elation group has class 3 , while the tame group has class 2 ;

(vi) each elation group in $(v)$ has exponent 4. 
Proof. Suppose $G$ and $H$ are distinct elation groups for $\mathcal{S}^{x}$, and let $W(x)$ be the group of all whorls about $x$, so that $|W(x)|>|G|$. Let $p$ be the characteristic prime of $\mathcal{S}^{x}$, and suppose $p$ divides $[W(x): G]$. Since $s t$ is a $p$-power, a combination of Theorems 2.5 and 2.2 (recalling that $x$ is a regular point) yields that any element of $W(x)_{z}$ of order $p^{h}(h>0), z$ being any opposite point to $x$, fixes a subGQ of order $(t, t)$ pointwise, while $s=t^{2}$. By Lemma 5.1 of [28], $p=2$, and $[S(x): G]=2$, where $S(x)$ is a Sylow 2-sugroup of $W(x)$ containing $G$. Also, such a subGQ is isomorphic to $\mathcal{W}(t)$.

Now suppose $p$ does not divide $[W(x): G]$. Then all ("full") elation groups about $x$ are Sylow $p$-subgroups in $W(x)$, so by Sylow's Theorem

$$
[W(x): G] \geq p+1
$$

The Sylow subgroups are conjugate, so they all contain $\mathbb{S}$ (as usual the group of symmetries about $x$ ). Now consider the action of $W(x)$ on the set $T$ of spans of noncollinear points in $x^{\perp}-$ the kernel of this action is $\mathbb{S}$. If $\theta \in W(x) / \mathbb{S}$ fixes distinct elements of $T$, then either $\theta$ is trivial, or it fixes a subplane of order $t$ of the dual net $\mathcal{N}_{x}^{D}$ (in which case $s=t^{2}$ ), cf. [38].

Suppose this latter case cannot occur for any $\theta$; then $(W(x) / \mathbb{S}, T)$ is a Frobenius group, and if $F$ is the Frobenius kernel, then $F^{\prime}$, the unique subgroup of $W(x)$ containing $\mathbb{S}$ which projects $F$ onto $W(x) / \mathbb{S}$, is a normal Sylow $p$-subgroup of $W(x)$, contradiction. Whence this situation does not occur.

Now assume some $\theta$ fixes a subplane of order $t$ elementwise, and choose $\theta$ in such a way that it is contained in $W(x)_{z}, z$ a point opposite $x$. (This choice can be done without loss of generality since $\mathcal{S}^{x}$ is an EGQ.) Then the fixed elements structure of $\theta$ is again a subGQ of order $t$, and $\theta$ is henceforth an involution in $\operatorname{Aut}\left(\mathcal{S}^{x}\right)$. Whence by Lemma 5.1 of [28], $\operatorname{char}\left(\mathcal{S}^{x}\right)$ is 2, and $[W(x): G]=2$, contradicting our assumption.

This proves (i) and (ii).

The remaining cases are (obiously) less trivial. We proceed as follows.

First let $\left\{\theta_{i}\right\}$ be the set of all involutions in $W(x)$ that fix a $\mathcal{W}(t)$-subGQ through $x$ pointwise, and define the injective map

$$
\Theta:\left\{\theta_{i}\right\} \hookrightarrow 2^{\mathcal{S}^{x}}: \theta \longmapsto \mathcal{S}_{\theta},
$$

which associates to each element of $\left\{\theta_{i}\right\}$ its fixed elements structure. Here, $2^{\mathcal{S}^{x}}$ is the set of all subgeometries of $\mathcal{S}^{x}$. Any two of these involutions are clearly conjugate (in each Sylow 2subgroup of $W(x)$ such involutions are easily seen to be conjugate). Let $K$ be a Sylow 2-subgroup of $W(x)$ containing some prescribed elation group of $\mathcal{S}^{x}$, and let $\left\{\theta_{i}\right\}_{K}$ denote $\left\{\theta_{i}\right\} \cap K$ - note that this set is not empty. We are interested in how distinct subGQs associated to elements of $\left\{\theta_{i}\right\}_{K}$ can intersect (which will enable us to detect part of the structure of the STGQ). Theoretically, ideal subGQs $\mathcal{S}^{\prime}$ and $\mathcal{S}^{\prime \prime} \neq \mathcal{S}^{\prime}$ could intersect in any of the following ways (cf. [41, Lemma 4.2.5] for a proof):

(1) $\mathcal{S}^{\prime} \cap \mathcal{S}^{\prime \prime}$ is a set of $t^{2}+1$ pairwise nonconcurrent lines of $\mathcal{S}^{\prime}$ and $\mathcal{S}^{\prime \prime}$;

(2) $\mathcal{S}^{\prime} \cap \mathcal{S}^{\prime \prime}$ consists of a line $L$ of $\mathcal{S}^{\prime}$ (and $\mathcal{S}^{\prime \prime}$ ), together with all points of $\mathcal{S}^{\prime}$ (and $\mathcal{S}^{\prime \prime}$ ) incident with this $L$, and all lines of $\mathcal{S}^{\prime}$ (and $\mathcal{S}^{\prime \prime}$ ) incident with these points;

(3) $\mathcal{S}^{\prime} \cap \mathcal{S}^{\prime \prime}$ is a GQ of order $(1, t)$.

Case (3) cannot occur by the twisting argument of the preceding section: since $\Theta$ is injective, $K_{z}$, $z$ being a hypothetical point of $\mathcal{S}^{\prime} \cap \mathcal{S}^{\prime \prime}$ not in $x^{\perp}$, must contain distinct involutions, contradicting its size. Case (1) can evidently not occur, so (2) remains. It follows easily that 


$$
\left|\left\{\theta_{i}\right\}_{K}\right|=\left|\operatorname{Im}(\Theta)_{K}\right|=t^{2}
$$

(It is clear what we mean with $\operatorname{Im}(\Theta)_{K}$.)

Now, following an idea of [44], define an incidence structure $\Pi(K)$ as follows.

- PoINTs are the $t^{2}$ subGQs of $\operatorname{Im}(\Theta)_{K}$ (“ $K$-subGQs").

- LINES are the point sets $\mathcal{S}^{\prime \prime} \cap M$, where $\mathcal{S}^{\prime \prime} \in \operatorname{Im}(\Theta)_{K}$ and $M$ a line incident with $x$.

- INCIDENCE is inverse containment.

Two distinct $K$-subGQs intersect in the $t+1$ points on some line through $x$, together with all lines on these points. So in $\Pi(K)$, two distinct points are incident with exactly one line. Let $\mathcal{S}^{\prime \prime}$ be a point, and $N$ be a line not containing $\mathcal{S}^{\prime \prime}$ in $\Pi$. Suppose $R I x$ is the line which contains the point set $N$ in $\mathcal{S}$. Then $\mathcal{S}^{\prime \prime} \cap R$ defines the unique line of $\Pi$ parallel to $N$ and containing $\mathcal{S}^{\prime \prime}$. It follows that $\Pi$ is an affine plane of order $t$. Since $K$ induces a translation group on $\Pi(K)$, the following properties hold:

(a) if $k \in K$ fixes a $K$-subGQ, it fixes all $K$-subGQs;

(b) by the previous property, $K_{\mathcal{S}^{\prime}}$ is a normal subgroup of $K$ for any $K$-subGQ $\mathcal{S}^{\prime}$, and $K / K_{\mathcal{S}^{\prime}}$ precisely is the translation group of $\Pi(K)$;

(c) $[K, K] \leq K_{\mathcal{S}^{\prime}}$.

By (c) we have

$$
[[K, K],[K, K]] \leq\left[K_{\mathcal{S}^{\prime}}, K_{\mathcal{S}^{\prime}}\right] \leq K_{\left[\mathcal{S}^{\prime}\right]}
$$

the latter being a group of order 2 . (Remark that $\mathcal{W}(t)$ is a TGQ since $\operatorname{char}\left(\mathcal{S}^{x}\right)=2$, so $K_{\mathcal{S}^{\prime}} / K_{\left[\mathcal{S}^{\prime}\right]}$ is abelian.) Whence if $H$ is an elation group w.r.t. $x$ in $K$, we have

$$
[[H, H],[H, H]] \leq K_{\left[\mathcal{s}^{\prime}\right]} \cap H=\{\mathrm{id}\} .
$$

So the solvability length of $H$ is at most (and then precisely) 2. We need to determine the nilpotency class. Note first that since $[H, H] \leq H_{\mathcal{S}^{\prime}},[H, H]$ is in the translation group of any of the $\mathcal{W}(t)$ 's earlier referred to, and in particular $[H, H]$ is abelian. Fix such a subGQ $\mathcal{S}^{\prime} \cong \mathcal{W}(t)$, and let $T$ be its translation group w.r.t. $x$. Let $\gamma \in T^{\times}$; then $\gamma$ fixes some line $L \mathrm{I} x$ pointwise in $\mathcal{S}^{\prime}$. Since $H_{\mathcal{S}^{\prime}} \unlhd H, \gamma$ has this property in each element of $\mathcal{S}^{\prime H}$, so $L$ is also fixed pointwise in $\mathcal{S}^{x}$ by $\gamma$. So $T$ satisfies (*) as a subgroup of $\operatorname{Aut}\left(\mathcal{S}^{x}\right)$.

Next we show that if $U \mp x, U \in \mathcal{S}^{\prime}, H_{U}$ fixes $\operatorname{proj}_{U} x$ linewise. For elements of $H_{\mathcal{S}^{\prime}} \cap H_{U}$ this is clear, so we consider an element $\zeta$ of $H_{U}$ not fixing $\mathcal{S}^{\prime}$. Then $H_{U}$ fixes the line $B$ of $\Pi(K)$ containing $\operatorname{proj}_{U} x=: u$ (as a point set). As a point set, $B$ contains $t$ points of $x u$, so $H_{U}$ fixes at least one point $u^{\prime} \neq u$ in this set. In fact, $H_{U}$ fixes all points of $B$. To see this, let $\gamma \in H_{U}$ send $z$ to $z^{\prime} \neq z$, both points in $B$. As $\gamma^{2}$ is a symmetry of $\mathcal{S}^{\prime}$ with axis $x u, \gamma$ acts as an involution on the affine lines which contain a point of $B$. Let $Z \mathrm{I} z$ be such an affine line, and put $Z^{\prime}:=Z^{\gamma}$. Let $v$ be any element of $H_{\mathcal{S}^{\prime}}$ which sends $Z$ to $Z^{\prime}$; it is also an involution, and fixes some line $F$ incident with $x$ and different from $x u$ pointwise. It follows that $[\gamma, v]=\mathrm{id}$ (as $Z$ is fixed by $[\gamma, v]$, and $F$ pointwise). This means that $\gamma$ must fix the line $U^{v} \not U$. It is easy to see that this contradicts the fact that $\left\{U, U^{v}\right\}$ is a regular pair of lines in each of the $K$-subGQs on $B$, together with the fact that any triad of lines in $\mathcal{S}^{x}$ has precisley $t+1$ centers. So indeed, $H_{U}$ fixes all points in $B$.

Now take any $K$-subGQ on $B$, say $\widehat{\mathcal{S}}$, and consider an affine line $C$ in $\widehat{\mathcal{S}}$ which is not concurrent with $x u$. Then $\left[\zeta, H_{C} \cap H_{\mathcal{S}^{\prime}}\right]=\mathbb{S}$, for clearly $\left[\zeta, H_{C} \cap H_{\mathcal{S}^{\prime}}\right] \leq \mathbb{S}$, and $\zeta^{a}=\zeta^{b}$ with $a, b \in H_{C} \cap H_{\mathcal{S}^{\prime}}$ would imply that $\zeta^{a b}=\zeta$, which we have already seen not to be possible.

Note that for any $k \in H_{C} \cap H_{\mathcal{S}^{\prime}}$ we have

$$
\left[\zeta, \zeta^{k}\right]=\zeta^{-2}\left[\zeta^{-1}, k\right] \zeta^{2}[\zeta, k]=\left[\zeta^{-1}, k\right][\zeta, k]=\left([k, \zeta]\left[k, \zeta^{-1}\right]\right)^{-1}=\mathrm{id} .
$$


(We use the fact that after the first equation symbol all elements are in $H_{\mathcal{S}^{\prime}}$, so commute with each other. Also, one notes that $[k, \zeta]$ and $\left[k, \zeta^{-1}\right]$ are inverses of each other, as $\zeta$ and $\zeta^{-1}$ have the same action on $(x u)^{\perp} \cap \mathcal{S}^{\prime}$, and so both commutators yield the same symmetry in $\mathbb{S}$.)

So indeed $\left\langle\zeta^{k} \mid k \in H_{C} \cap H_{\mathcal{S}^{\prime}}\right\rangle$ is an abelian group containing $\mathbb{S}$, which is what we wanted to prove.

Now consider $[[H, H], H]$; it is a subgroup of $T=H_{\mathcal{S}^{\prime}}$, so it also satisfies $\left(^{*}\right.$ ) (as does $[T, H]$ ). We have a look at two types of commutators in $[T, H]$.

- Type $[\alpha, \beta]$, with $\alpha \in T$ fixing $L I x$ pointwise, and $\beta \in H$ fixing $y \sim x \neq y$ linewise, $y \pm L$. Such a commutator is a symmetry about $x$.

- Type $[\alpha, \beta]$, with $\alpha \in T$ fixing $L I x$ pointwise, and $\beta \in H$ fixing $y \sim x \neq y$ linewise, $y \mathrm{I} L$. Then $[\alpha, \beta]$ is a root-elation in $\mathcal{S}^{\prime}$, so it is a symmetry about $L$ in $\mathcal{S}^{\prime}$. It easily follows that it also must be a symmetry about $L$ in $\mathcal{S}^{x}$, which contradicts $s=t^{2}$ and [27, 8.1.2].

Since $H$ is generated by the set $V$ of elements $\beta$ as above, we have that $[[H, H], H] \leq[T, H] \leq \mathbb{S}$. Since again $H$ is generated by $V$, the nilpotency class of $H$ is thus at most 3 .

Class 1 is not possible since $t<s$, an obstruction for TGQs.

Now suppose the class of $H$ is precisely 2 , so that $\mathbb{S} \leq Z(H)$. Then in $H$, all Moufang conditions are satisfied w.r.t. $x$ and $H$ is generated by the as such defined root groups (cf. [44]), so that $H$ is tame, and unique. (Note that, conversely, if $H$ would be tame, then clearly $\mathbb{S} \leq Z(H)$, and $H$ is of class 2.)

Next suppose $H$ to have class 3 - not all local Moufang conditions in $H$ w.r.t. $x$ are satisfied. We need to show that all i-roots and dual i-roots are Moufang, so that there indeed exists a tame elation group, which is then unique by the above. So let $U \nsim x$ be a line, and suppose $V=\operatorname{proj}_{x} U$. Let $S$ be a Sylow 2-subgroup in $W(x)$. Construct the subGQ-plane $\Pi(S)$ w.r.t. $S$, and let $B$ be the line containing $V \cap U$ as a point set. Then $S_{U}$ has order $2 t^{2}$, and $S_{U}$ acts on $\Pi(S)$. Note the following facts:

- $S_{U}$ fixes the point set corresponding to $B$ elementwise, and fixes each line incident with $V \cap U$;

- any element of $S_{U}$ which fixes a subGQ corresponding to a point incident with $B$ in $\Pi(S)$, either fixes it pointwise or induces a translation;

- if an element of $S_{U}$ fixes one, and then all, subGQs corresponding to $\Pi(S)$, then, since a translation in $\mathcal{W}(t)$ always fixes some line pointwise, it fixes $V$ pointwise if (and only if) it is an elation with center $x$.

It follows that $\left|S_{U} / N\right|=2$, with $N$ the kernel of $S_{U}$ in its action on the points of $V$. So if $z \mathrm{I} V, z \notin B \cup\{x\}$, then $S_{U, z}$ is a group of size $t^{2}$ which fixes each point incident with $V$. So $(V \cap U, V, x)$ is a Moufang i-root with root group $S_{U, z}$. This proves (iv)-(v).

(vi) follows easily from the fact that, if $\alpha \in H$, with $H$ some full elation group w.r.t. $x, H$ acting on the appropriate subGQ-plane (corresponding to a Sylow 2-subgroup containing $H$ ), then $\alpha^{2}$ is the identity on the plane. So it fixes the subGQs in question, and hence it either is an involution fixing one of these subGQs pointwise (but then it is not in $H$ ), or it is a translation in one of these subGQs.

Corollary 11.2. $\mathbb{S} \leq Z(H)$ for any full elation group with center $x$.

Proof. Let $\widehat{E}$ be the tame elation group. Then $\widehat{E}$ is generated by the root-elations of $\mathcal{S}^{x}$ with i-root containing $x$. But each such elation commutes with any element of $\mathbb{S}$. So $\mathbb{S} \leq Z(\widehat{E})$. For non-tame elation groups, the argument is similar (replacing root groups by groups of the form $H_{U}$, 
$U \nsim x$, and remarking that $H_{U}$ fixes $\operatorname{proj}_{U} x$ linewise).

Corollary 11.3. Let $H$ be as in the theorem (a non-tame full elation group). Let $\gamma \in H \backslash \mathbb{S}$ fix points besides $x$. Then we have the following possibilities.

(i) $\gamma$ fixes precisely $t+1$ points, and $\gamma \notin T$. Also, $\gamma$ fixes precisely one point $\neq x$ linewise.

(ii) $\gamma$ fixes $t^{2}+1$ points, and $\gamma \in T$. Also, $\gamma$ fixes $t^{2}$ affine lines.

Proof. By the previous theorem, we know that $H$ arises from twisting the unique tame elation group $L$ by some involution $\theta$ which fixes some ideal $\mathcal{W}(t)$-subGQ elementwise. So $\gamma=\ell$ or $\gamma=\theta \ell$, with $\ell \in L$. This immediately gives us the information concerning the fixed points in (i) and (ii). If we are in case (i), then $\gamma \notin T$ (as we have already seen), and by our first fixed point theorem, we also know that $\gamma$ must have fixed affine lines. The $t$ fixed points different from $x$ are all in some $\Pi(K)$-line (cf. the proof of the previous theorem), and by the same proof, all fixed lines must be incident with the same point. Clearly, this point is fixed linewise (by the fixed point theorem, or the fact that $\mathbb{S} \leq Z(H)$ ).

Now suppose that $\gamma$ fixes the $t^{2}+1$ points on some line incident with $x$. By the fixed point theorem, $\gamma$ also fixes precisely $t^{2}$ affine lines. We have seen that if $\gamma \notin T$, all these lines must contain a point of one and the same $\Pi(K)$-line. But we have also seen that such $\gamma$ cannot exist.

The next corollary is now immediate.

Corollary 11.4. Let $H$ be as in the previous corollary. Then the $i$-root elations and dual $i$-root elations generate $T$.

We are ready to obtain the main result of this section.

Theorem 11.5 (Payne's question). If $\mathcal{S}^{x}$ is as in the previous theorem, it is isomorphic to $\mathcal{H}\left(3, t^{2}\right)$.

Proof. Let $H$ be as in the previous theorem. Let $\mathcal{S}^{\prime}$ be a $\mathcal{W}(t)$-subGQ as above, and let $\mathcal{S}^{\prime \prime}$ be another one in the same $H$-orbit. Let $U \in \mathcal{S}^{\prime \prime}$ be an affine line; it is exterior to $\mathcal{S}^{\prime}$, so it is concurrent with $t^{2}+1$ lines $T_{U}$ of $\mathcal{S}^{\prime}$ which partition its points (cf. the dual of Theorem 2.1). Define $A:=H_{U} \cap H_{\mathcal{S}^{\prime}}$ - this subgroup of $H$ of size $t$ stabilizes $\mathcal{S}^{\prime}$, and also the set $T_{U}$. As $A$ fixes $U$, Corollary 11.3 readily implies that $[U]:=\operatorname{proj}_{x} U$ is fixed pointwise by $A$. Dualizing, and noting that $\mathcal{W}(t)$ is self-dual as $t$ is even [27, 3.2.1], $T_{U}$ becomes an ovoid $\mathcal{O}$ of $\mathcal{S}^{\prime D} \cong \mathcal{W}(t)$ (which is also an ovoid in the ambient space of $\mathcal{W}(t)$ - see [33]) fixed by the group $A ; A$ also fixes a flag $(X,[u])$ (corresponding to $(x,[U])$ ) "elementwise" (all the points on $X$ and all the lines on $u$ ). Let $\mathcal{O}^{\prime}$ be any $A$-orbit on $\mathcal{O}$, different from $\{[u]\}$; each of its points is collinear with one and the same point of $X$, so the points of $\mathcal{O}^{\prime}$, together with $X$, generate a plane in the ambient $\mathbf{P G}(3, t)$ of $\mathcal{W}(t)$. It easily follows that $\mathcal{O}^{\prime}$ is a translation oval, and so the $t A$-orbits on $\mathcal{O}$ different from $\{[u]\}$ define a pencil of translation ovals with axis $X$, which lies in the tangent plane of $\mathcal{O}$ at $[u]$. By [29], it follows that $\mathcal{O}$ either is an elliptic quadric or a Suzuki-Tits ovoid. The main theorem of [6] now implies that $\mathcal{S}^{x} \cong \mathcal{H}\left(3, t^{2}\right)$.

We will leave the next corollary (of the proof) to the reader.

Corollary 11.6. Let $\left(\mathcal{S}^{x}, H\right)$ be a finite EGQ of order $(s, t)$, and let $\mathcal{S}^{\prime}$ be a proper ideal subquadrangle of order $(t, t)$ containing $x$. If $t$ is even and $\mathcal{S}^{\prime}$ is fixed pointwise by some involutory automorphism of $\mathcal{S}^{x}, \mathcal{S}^{x} \cong \mathcal{H}\left(3, t^{2}\right)$.

(First show that $\left(\mathcal{S}^{x}, H\right)$ is an STGQ, Then apply the previous theory.)

It might be in reach to conclude the same result as in the previous theorem, but with "subGQ of order $(t, t)$ plus involution fixing it pointwise" replaced by " $\mathcal{W}(t)$-subGQ." I will come back to this issue in a future paper. 


\section{PART V}

\section{GENERIC STGQs}

\section{GENERIC STGQS}

When an STGQ $\left(\mathcal{S}^{x}, H\right)$ satisfies $(*)$, or equivalently when $T=H / \mathbb{S}$ is abelian, one easily observes that when $\left(\mathcal{F}, \mathcal{F}^{*}\right)$ is the associated Kantor-family, and $A, B$ are distinct members of $\mathcal{F}$, that if $U \leq A$ and $V \leq B$, we have

$$
\langle U, V\rangle=H \longmapsto U=A, \quad V=B .
$$

(If at least one of $U, V$ is a proper subgroup, $\langle U \mathbb{S} / \mathbb{S}, V \mathbb{S} / \mathbb{S}\rangle$ is a proper subgroup of $T$.) We will show that a generalization of this natural property suffices to classify the parameters of the generic case (that is, the case where $T$ is not abelian), and to even classify to some extent. So below, a generic $S T G Q$ is an STGQ for which $T$ is not abelian, or equivalently, for which (*) is not satisfied. In particular, such STGQs cannot be square STGQs.

More precisely, in a first instance our main objective is proving the following result (Theorem 12.1 below). Before stating it, we need a definition. Let $\left(\mathcal{S}^{x}, H\right)$ be as above, and let $\Phi:=\Phi(H)$ be the Frattini subgroup of $H$. Define a point-line geometry $\Gamma(\Phi)$ as follows. Its lines are the $\Phi$-orbits on the lines incident with $x$ (where the trivial orbit $\{x\}$ is excluded); its points are the $\Phi$-orbits in the affine point set of $\mathcal{S}^{x}$. A point $u$ is incident with a line $V$ if at least one $\mathcal{S}^{x}$-point of the orbit $V$ is collinear with some point of the orbit $u$. If $u$ then is incident with $V$, it is easy to see that $V$ is surjectively projected on $u$, so that for each $L I x$, a $\Gamma(\Phi)$-point is incident with precisely one line which is a $\Phi$-orbit on $L$. So each $\Gamma(\Phi)$-point is incident with precisely $t+1 \Gamma(\Phi)$-lines.

Theorem 12.1. Let $\left(\mathcal{S}^{x}, H\right)$ be a generic $S T G Q$ of order $(s, t)$, with Kantor family $\left(\mathcal{F}, \mathcal{F}^{*}\right)$. Suppose that the projection lemma (cf. the next subsection) holds. Suppose also that at least one of the following properties is satisfied.

(a) $\Gamma(\Phi)$ is a dual partial linear space.

(b) For each $A \neq B \in \mathcal{F}$, we have that if $K$ is a maximal subgroup of $H$ which does not contain $A$, then

$$
\langle A \cap K, B\rangle \neq H .
$$

(This is the generalization of the aforementioned natural property.)

Then the Frattini subgroup $\Phi(H)$ is the elation group of a subSTGQ of order $t$, so that $s=t^{2}$.

The way to construct ideal subquadrangles goes as follows. Let $\left(\mathcal{K}, \mathcal{K}^{*}\right)$ be a Kantor family of type $(s, t)$ in the group $G$, and put $\mathcal{F}:=\mathcal{K} \cup \mathcal{K}^{*}$. A nontrivial subgroup $X$ of $G$ is an $\mathcal{F}$-factor of $G$ if

$$
(U \cap X)(V \cap X)=X \text { for all } U, V \in \mathcal{F} \text { satisfying } U V=G .
$$

Define $\mathcal{K}_{X}=\{U \cap X \mid U \in \mathcal{K}\}$ and $\mathcal{K}_{X}^{*}=\left\{U^{*} \cap X \mid U^{*} \in \mathcal{K}^{*}\right\}$. We say that $X$ is "of type $(\sigma, \tau)$ " if $|X|=\sigma^{2} \tau,|A \cap X|=\sigma$ and $\left|A^{*} \cap X\right|=\sigma \tau$ for all $A \in \mathcal{K}$ (and in [16] it is shown that such integers $\sigma, \tau$ always exist).

Theorem 12.2 (D. Hachenberger [16]). Let $X$ be an $\mathcal{F}$-factor of type $(\sigma, \tau)$ in $G$. Then necessarily one of the following cases occurs:

(a) $\sigma=1,|X|=\tau \leq t$ and $X$ is a subgroup of $\cap_{A \in \mathcal{K} A^{*} \text {; }}$

(b) $\sigma>1, \tau=t$ and $\left(\mathcal{K}_{X}, \mathcal{K}_{X}^{*}\right)$ is a Kantor family in $X$ of type $(\sigma, \tau)$. 
If we are in case (b) of Theorem 12.2, we call $X$ a thick $\mathcal{F}$-factor. An $\mathcal{F}$-factor $X$ in $G$ is normal if $X$ is a normal subgroup of $G$.

Theorem 12.3 (D. Hachenberger [16]). Let $G$ be a group of order $s^{2} t$ admitting a Kantor family $\left(\mathcal{K}, \mathcal{K}^{*}\right)$ of type $(s, t)$, with $s, t>1$, and having a normal $\mathcal{F}$-factor $X$ of type $(\sigma, \tau)$ with $\tau=t$. Then one of the following cases occurs:

(a) $G$ is a group of prime power order;

(b) $\sigma>1,|G|$ has exactly two prime divisors, and $X$ is a Sylow subgroup of $G$ for one of these primes.

Once we have ideal subSTGQs, we can use our theory of square STGQs to search for more structure in the generic case.

The reader might want to keep the following standard and well-known properties in mind:

Lemma 12.4. Let $G$ be a finite group. Then the following are true.

(i) If $G$ is a p-group, then $\Phi(G)=[G, G] G^{p}$, where $G^{p}:=\left\langle g^{p} \mid g \in G\right\rangle$.

(ii) For general $G, \Phi(G)$ consists of the non-generating elements of $G$ (an element $g$ is nongenerating if for any generating set $\Omega$ of $G$ that includes $g, \Omega \backslash\{g\}$ also is a generating set).

(iii) For general $G, \Phi(G)$ is the intersection of all maximal subgroups in $G$.

Remark 12.5. In the course of the series, we will show that "most of the time," $H$ has exponent $p$, so that $\Phi(H)=[H, H]$. But when the exponent is 4 , it might happen that $|[H, H]|=t^{3} / 2-$ in fact this happens for the exotic elation groups of $H\left(3, q^{2}\right)$ with $q$ even.

12.1. Projection Lemma. Let $\left(\mathcal{S}^{x}, H\right)$ be an STGQ of order $(s, t)$. Let $T=H / \mathbb{S}$ be the translation group of the net $\Pi_{x}$. In this paragraph we wish to understand the extensions

$$
\mathrm{id} \longmapsto M \cap \mathbb{S} \longmapsto M \longmapsto T \longmapsto \mathrm{id},
$$

with $M \leq H$. This will render us information about the Frattini subgroup of $H$.

The following lemma will be crucial (it is Theorem 5.2 (iv) applied to STGQs with (*)).

Lemma 12.6. Suppose $\mathcal{S}^{x}$ satisfies $(*)$. If $s=t$ is odd, or $s \neq t$, any element $\alpha$ of $\left(\cup_{A \in \mathcal{F}} A^{*}\right) \backslash \mathbb{S}$ maps some point of $\mathcal{P} \backslash x^{\perp}$ to a collinear point.

Proof. Suppose $\alpha \notin \mathbb{S}$ does not have this property. Applying Benson's lemma (while using (*)), we obtain

$$
(t+1)(s+1)+t s \equiv s t+1 \bmod s+t,
$$

so that $s+t$ divides $s t$, contradiction.

Corollary 12.7. If $s=t$ is odd, or if $s \neq t$ and $T$ is abelian, the previous lemma applies.

Proof. By assumption, $T$ is abelian, so $(*)$ holds for $\mathcal{S}^{x}$ by Theorem 5.1 .

Suppose $\mathcal{S}^{x}$ has the property that any element $\alpha$ of $\left(\cup_{A \in \mathcal{F}} A^{*}\right) \backslash \mathbb{S}$ maps some point of $\mathcal{P} \backslash x^{\perp}$ to a collinear point. Suppose that $(*)$ holds. Let $M$ be a subgroup as above, and note that without of loss of generality, we can suppose that $M$ is maximal in $H$. Let $\left(\mathcal{F}, \mathcal{F}^{*}\right)$ be the Kantor family associated to $\left(\mathcal{S}^{x}, H\right)$. Note that there must be some $A \in \mathcal{F}$ not contained in $M$, since $\langle A| A \in$ $\mathcal{F}\rangle=H$. Note that $[H: M]=p=\left[A^{*}: A^{*} \cap M\right]$, where st is a power of the prime $p$. Let $L \mathrm{I} x$ be such that $L \neq[A]$, so that there are $p(A \cap M)$-orbits on $L \backslash\{x\}$. On the other hand, $M$ acts transitively on $L \backslash\{x\}$ since $M$ projects on $T$. Moreover, since $\left[A^{*}: A^{*} \cap M\right]=p$ and 
$\mathbb{S} \not \leq M, A^{*} \cap M$ also acts transitively on $L \backslash\{x\}$. Let $\beta \in M \cap A^{*}$ be such that it does not fix all $(A \cap M)$-orbits on $L \backslash\{x\}$. Then $\beta$ fixes some line $U \notin x$ (since it maps some affine point to a collinear point), and clearly $U \sim[A]$. Now $H_{U}$ is conjugate to $A$ by some element $\gamma$ of $H_{[L]}$, so $\beta$ is conjugate to some element of $A$ with the same action on $L$. But as $M$ is a normal subgroup of $H, \beta \in M$, so that $A \in M$, contradiction.

Theorem 12.8 (Projection Lemma). The only extension of $T$ in $H$ is $H$ itself, if one of the following properties is satisfied.

(i) $s=t$ is odd.

(ii) $s \neq t$, and $\mathcal{S}^{x}$ satisfies $(*)$.

Remark 12.9 (Even case). When $s=t$ is even, Theorem 12.8 cannot work in general. For, let $\mathcal{S}^{x}$ be a TGQ of order $2^{h}$, so that $x$ is a regular point. Take any two distinct elements $A, B$ of the associated Kantor family. Then $A B=\langle A, B\rangle$, and $A B \mathbb{S} / \mathbb{S}=T$.

Let $M$ be maximal in $H$. By Theorem $12.8, M /(M \cap \mathbb{S})$ is an index $p$-group in $T$, so that $\mathbb{S} \leq M$. The following corollary is in some sense complementary to what we will encounter in the abelian factor section, later in part 2 of the series.

Corollary 12.10 (Structure of abelian $T)$. Let $\left(\mathcal{S}^{x}, H\right)$ satisfy one of $(i)-(i i)$ of the previous theorem.

(i) If $A, B \in \mathcal{F}$ are distinct, $\langle A, B\rangle=H$. Whence $\mathbb{S} \leq \Phi(H)$.

(ii) If $T$ is abelian, $\mathbb{S}=[H, H]$, and every element of $\mathbb{S}$ is a commutator.

(iii) $\Phi(H)=\mathbb{S}=[H, H]$ if and only if $T$ is elementary abelian (so in particular, if $s=t$ is odd).

Proof. It is clear that $\langle A, B\rangle \mathbb{S} / \mathbb{S}=T$, so $\langle A, B\rangle=H$ by Theorem 12.8 . Each generating set $\Omega$ of $H$ still generates an extension of $T$ if all elements of $\mathbb{S}$ are removed from $\Omega$, as $\mathbb{S}$ acts trivially on $x^{\perp}$. By Lemma 12.4 (ii), $\mathbb{S} \leq \Phi(H)$. This proves (i).

Now let $T$ be abelian; then $[H, H] \leq \mathbb{S}$. Let $\gamma \in \mathbb{S}^{\times}$, and let $U \mp x$ be arbitrary. Let $\xi$ be an element of $H \backslash \mathbb{S}$ mapping $U$ to $U^{\gamma}$; then $\xi$ fixes some affine line $U^{\prime} \neq U$ which meets $\operatorname{proj}_{x} U$. Let $\beta \in H$ map $U^{\prime}$ to $U$. Then $[\beta, \xi]$ maps $U$ to $U^{\gamma}$, while being a symmetry. It follows that $[H, H]=\mathbb{S}$ and that every element of the latter is a commutator, whence (ii).

Both groups $[H, H], \mathbb{S}$ coincide with $\Phi(H)$ if $T$ is elementary abelian, by Lemma 12.4(i). Vice versa, if $T$ is elementary abelian,

$$
\bigcap_{Y \leq T \text { maximal }} Y=\{\mathrm{id}\},
$$

so that $\Phi(H)=\mathbb{S}$ by Lemma 12.4 (iii). This proves (iii).

The following lemma follows in the same way as the Projection Lemma.

Theorem 12.11 (Projection Lemma - general form). Suppose $\left(\complement^{x}, H\right)$ is an STGQ of order $(s, t)$, and let $\left(\mathcal{F}, \mathcal{F}^{*}\right)$ be the associated Kantor family. Let one of the following properties be satisfied.

(i) $s=t$ is odd.

(ii) $s \neq t$, and $\mathfrak{S}^{x}$ satisfies $(*)$.

Let $N$ be a normal subgroup of $H, L \mathrm{I} x$, and $O$ an $N$-orbit in $L \backslash\{x\}$. If $O$ is also an $\left(A^{*} \cap N\right)$ orbit for $A^{*} \in \mathcal{F}^{*}$, then $O$ is an A-orbit.

For a generic STGQ $\left(\mathcal{S}^{x}, H\right)$, we say that the projection lemma holds if the following short exact sequence

$$
\mathrm{id} \longmapsto M \cap \mathbb{S} \longmapsto M \longmapsto T \longmapsto \mathrm{id},
$$


with $M \leq H$, only has solutions for $M=H$.

Lemma 12.12. If the projection lemma holds for the generic $S T G Q\left(\mathcal{S}^{x}, H\right)$, then $\mathbb{S} \leq \Phi(H)$.

Proof. $\quad$ Let $M$ be any maximal subgroup of $H$; then

$$
\frac{|H|}{\operatorname{char}\left(\mathcal{S}^{x}\right)}=|M|=|M \cap \mathbb{S}| \times|M /(M \cap \mathbb{S})|
$$

so that the projection lemma implies that $\mathbb{S} \leq M$ (another way to prove this is as in Corollary $12.10(\mathrm{i})$ ).

12.2. Generic STGQs with (b). In this subsection we suppose that $\left(\mathcal{S}^{x}, H\right)$ is a generic STGQ of order $(s, t)$ with Kantor family $\left(\mathcal{F}, \mathcal{F}^{*}\right)$, so that $s \neq t$. For the entire subsection, we assume to be in part (b) of the theorem. We may suppose w.l.o.g. that $\mathbb{S}$ is contained in $\Phi=\Phi(H)$. Let $\mathbb{S} \leq G \leq H$, and $A, B \in \mathcal{F}$ with $A \neq B$. We say that $G$ satisfies (F) "at $A$ with respect to $B^{*}$ " if

$$
G=(G \cap A)\left(G \cap B^{*}\right) .
$$

Note that this property is symmetric in $A$ and $B$. If $G$ has (F) at $A$ w.r.t. every $B^{*} \in \mathcal{F}^{*} \backslash\left\{A^{*}\right\}$, by definition $G$ satisfies (F) at $A$.

Let $A \in \mathcal{F}$. Define $\mathcal{R}_{A}$ to be the set of maximal subgroups of $H$ that contain $A$; note that for $R \in \mathcal{R}_{A}$, (F) is satisfied at $A$ w.r.t. any element of $\mathcal{F}^{*} \backslash\left\{A^{*}\right\}$. Let $\mathcal{K}_{A}:=\cap_{C \in \mathcal{R}_{A}} C$. Then the reader verifies that $\mathcal{K}_{A}$ also satisfies (F) at $A$ w.r.t. any element of $\mathcal{F}^{*} \backslash\left\{A^{*}\right\}$. (If $A \leq L \leq H, L$ has (F) at $A$ w.r.t. any such element.)

Lemma 12.13. Let $A, B$ be different elements in $\mathcal{F}$. If $K$ is a maximal subgroup in $H$ not containing $B$, then there is a maximal subgroup $K^{\prime}$ containing $A$ for which $K^{\prime} \cap B=K \cap B$. In particular, we have that $\mathcal{K}_{A} \cap B \leq K \cap B \leq K$ for all maximal subgroups $K$. So $\Phi \cap B=\mathcal{K}_{A} \cap B$ if $A \neq B$ are elements in $\mathcal{F}$, and whence for a fixed $B \in \mathcal{F}, \mathcal{K}_{A} \cap B$ is independent of the choice of $A \neq B$ in $\mathcal{F}$.

Proof. As $\langle A, B \cap K\rangle \neq H$, there is a maximal subgroup $K^{\prime}$ containing $\langle A, B \cap K\rangle$. Clearly, $K^{\prime} \cap B=K \cap B$ (as otherwise $B \leq K^{\prime}$, contradicting the projection lemma).

Lemma 12.13 implies that for any $C, D \neq C \in \mathcal{F}$, and any $A \in \mathcal{F}$ (where $A=C$ or $A=D$ are allowed),

$$
A \cap \Phi=A \cap \mathcal{K}_{C}=A \cap \mathcal{K}_{D}=A \cap \mathcal{K}_{C} \cap \mathcal{K}_{D}
$$

This property is obtained in an alternative and slightly more general way in the next two lemmas.

Lemma 12.14. Let $\mathbb{S} \leq L, M \leq H$, with $L$ and $M$ normal subgroups of $H$. Suppose that $L$ and $M$ have $(F)$ at $A$ w.r.t. $B^{*}$ (and so also at $B$ w.r.t. $A^{*}$ ). Then $L \cap M$ also has $(F)$ at $A$ w.r.t. $B^{*}$.

Proof. We have that

$$
|L \cap M|=\frac{|L| \times|M|}{|L M|}=\frac{\left(|A \cap L| \times\left|L \cap B^{*}\right|\right) \times\left(|A \cap M| \times\left|B^{*} \cap M\right|\right)}{|L M|} .
$$

As $|L \cap M \cap A|=\frac{|L \cap A| \times|A \cap M|}{|(L \cap A)(A \cap M)|}$ and $\left|L \cap M \cap B^{*}\right|=\frac{\left|L \cap B^{*}\right| \times\left|B^{*} \cap M\right|}{\left|\left(L \cap B^{*}\right)\left(B^{*} \cap M\right)\right|}$, it follows that

$$
|L \cap M|=\left(|L \cap M \cap A| \times\left|L \cap M \cap B^{*}\right|\right) \times \frac{|(L \cap A)(A \cap M)| \times\left|\left(L \cap B^{*}\right)\left(B^{*} \cap M\right)\right|}{|L M|}
$$


Since $|L \cap M| \geq|L \cap M \cap A| \times\left|L \cap M \cap B^{*}\right|$, we have that

$$
\frac{|(L \cap A)(A \cap M)| \times\left|\left(L \cap B^{*}\right)\left(B^{*} \cap M\right)\right|}{|L M|} \geq 1 .
$$

On the other hand, $(L \cap A)(A \cap M) \subseteq L M \cap A$ and $\left(L \cap B^{*}\right)\left(M \cap B^{*}\right) \subseteq L M \cap B^{*}$, while $(L M \cap A)\left(L M \cap B^{*}\right) \subseteq L M$. It follows that

$$
\frac{|(L \cap A)(A \cap M)| \times\left|\left(L \cap B^{*}\right)\left(B^{*} \cap M\right)\right|}{|L M|}=1,
$$

so that $|L \cap M|=|L \cap M \cap A| \times\left|L \cap M \cap B^{*}\right|$.

Lemma 12.15. Let $A, B(\neq A) \in \mathcal{F}$. Then $\mathcal{K}_{A} \cap \mathcal{K}_{B}$ satisfies $(F)$ at $A$ w.r.t. $B^{*}$. It follows that $\Phi=\mathcal{K}_{A} \cap \mathcal{K}_{B}$ for any choice of different $A, B \in \mathcal{F}$.

Proof. Consider $\mathcal{K}_{A} \cap \mathcal{K}_{B}$. Then applying Lemma 12.14 with $L=\mathcal{K}_{A}$ and $M=\mathcal{K}_{B}$, we conclude that $\mathcal{K}_{A} \cap \mathcal{K}_{B}$ has (F) at $A$ w.r.t. $B^{*}$.

Noting that $\Phi \leq \mathcal{K}_{A} \cap \mathcal{K}_{B}=\left(\mathcal{K}_{A} \cap \mathcal{K}_{B} \cap A\right)\left(\mathcal{K}_{A} \cap \mathcal{K}_{B} \cap B^{*}\right)$, the fact that $\mathcal{K}_{A} \cap \mathcal{K}_{B} \cap A=$ $\Phi \cap A \leq \Phi$ and $\mathcal{K}_{A} \cap \mathcal{K}_{B} \cap B^{*}=\Phi \cap B^{*} \leq \Phi$, indeed implies that $\Phi=\mathcal{K}_{A} \cap \mathcal{K}_{B}$ for any choice of different $A, B \in \mathcal{F}$.

Corollary 12.16. Under the assumptions of this subsection, $\Phi=\Phi(H)$ is a normal $\mathcal{F}$-factor in $H$.

12.3. Generic STGQs with (a). Now we suppose that $\left(\mathcal{S}^{x}, H\right)$ is a generic STGQ of order $(s, t)$ with Kantor family $\left(\mathcal{F}, \mathcal{F}^{*}\right)$, and we assume to be in part (a) of the theorem. Let $A \neq B \in \mathcal{F}$, and suppose that $a b s=\phi$ with $a \in A, b \in B, s \in \mathbb{S}$, and $\varphi \in \Phi$. (Note that such $a, b, s$ always exist.) Then $a b=\phi s^{-1}=: \varphi \in \Phi$ by Lemma 12.12. As $a b \in \Phi, a b$ fixes all points and lines of $\Gamma(\Phi)$. As $\Phi \unlhd H, a$ fixes all lines of $\Gamma(\Phi)$ of which the corresponding orbits are subsets of $[B]$ (" $B$-lines"), and $b$ fixes all lines of $\Gamma(\Phi)$ of which the corresponding orbits are subsets of $[A]$ (" $A$-lines"). As $\Gamma(\Phi)$ is a dual partial linear space, and as each $\Gamma(\Phi)$-point is incident with one $A$-line and one $B$-line, it follows that $a$ and $b$ fix all $\Gamma(\Phi)$-points (and so all $\Gamma(\Phi)$-lines). One immediately deduces that $a \in \Phi$ and $b \in \Phi$. So for all $C \neq D \in \mathcal{F}$, we have shown that

$$
\Phi=(C \cap \Phi)\left(D^{*} \cap \Phi\right)=(D \cap \Phi)\left(C^{*} \cap \Phi\right) .
$$

Proposition 12.17. Under the assumptions of this subsection, $\Phi=\Phi(H)$ is a normal $\mathcal{F}$-factor for $H$.

12.4. Proof of Theorem 12.1. We have shown, under the assumptions of Theorem 12.1, that $\mathcal{F}_{\Phi}:=\{\Phi \cap A \mid A \in \mathcal{F}\}$ defines a Kantor family of type $\left(s^{\prime}, t\right)$ in $\Phi$, with $s>s^{\prime}>1$, and $\mathcal{F}_{\Phi}^{*}=\left\{\Phi \cap A^{*} \mid A \in \mathcal{F}\right\}$. Also, since $x$ is regular, we have that $s^{\prime}=t=\sqrt{s}$ by Theorem 2.2. 


\section{REFERENCES}

[1] J. L. Alperin And G. Glauberman. Limits of abelian subgroups of finite p-groups, J. Algebra 203 (1998), 533-566.

[2] J. BAmberg, S. P. Glasby And E. Swartz. AS-configurations and skew-translation generalised quadrangles, J. Algebra 421 (2015), 311-330.

[3] J. Bamberg, T. Penttila And C. Schneider. Elation generalized quadrangles for which the number of lines on a point is the successor of a prime, J. Aust. Math. Soc. 85 (2008), 289-303.

[4] S. G. Barwick, M. R. Brown and T. PentTila. Flock generalized quadrangles and tetradic sets of elliptic quadrics of PG(3, q), J. Combin. Theory Ser. A 113 (2006), 273-290.

[5] A. E. BROUWER. The complement of a geometric hyperplane in a generalized polygon is usually connected, Finite Geometry and Combinatorics (Deinze, 1992), Edited by F. De Clerck et al., London Math. Soc. Lecture Note Ser. 191, Cambridge University Press, Cambridge (1993), 53-57.

[6] M. R. Brown. Generalized quadrangles of order $\left(q, q^{2}\right), q$ even, containing $W(q)$ as a subquadrangle, Geom. Dedicata 56 (1995), 299-306.

[7] M. R. BRown. Generalized Quadrangles and Associated Structures, Ph.D. Thesis, University of Adelaide (1999), vii+141 pp.

[8] M. R. BRown. Projective ovoids and generalized quadrangles, Adv. Geom. 7 (2007), 65-81.

[9] X. ChEN. On the groups that generate skew translation generalized quadrangles, Unpublished Manuscript, 1990.

[10] X. Chen And D. FrohardT. Normality in a Kantor family, J. Combin. Theory Ser. A 64 (1993), 130-136.

[11] P. Deligne. Congruences sur le nombre de sous-groupes d'ordre $p^{k}$ dans un groupe fini, Bull. Soc. Math. Belg. 18 (1966), 129-132.

[12] S. De Winter And K. Thas. Generalized quadrangles admitting a sharply transitive Heisenberg group, Des. Codes Cryptogr. 47 (2008), 237-242.

[13] D. FrohardT. Groups which produce generalized quadrangles, J. Combin. Theory Ser. A 48 (1988), 139-145.

[14] D. GhinelLi. Characterization of some 4-gonal configurations of Ahrens-Szekeres type, European J. Combin. 33 (2012), 1557-1573.

[15] J. D. Gillam. A note on finite metabelian p-groups, Proc. Amer. Math. Soc. 25 (1970), 189-190.

[16] D. HaChenberger. Groups admitting a Kantor family and a factorized normal subgroup, Des. Codes. Cryptogr. 8 (1996), 135-143.

[17] D. Hachenberger. On the existence of translation nets, J. Algebra 152 (1992), 207-229.

[18] Y. Hiramine. Automorphisms of p-groups of semifield type, Osaka J. Math. 20 (1983), 735-746.

[19] N. L. Johnson, V. Jha And M. Biliotti. Handbook of Finite Translation Planes, Pure and Applied Mathematics 289 (Boca Raton), Chapman \& Hall/CRC, Boca Raton, FL, 2007.

[20] W. M. Kantor. Finite semifields, in: Finite Geometries, Groups, and Computation, 103-114, Walter de Gruyter GmbH \& Co. KG, Berlin, 2006.

[21] A. MANN. Groups with few class sizes and the centralizer equality subgroup, Israel J. Math. 142 (2004), 367-380.

[22] N. KnARR. Polar spaces, BLT-sets and generalized quadrangles, Adv. Geom. 8 (2008), 139-152.

[23] C. Parker And P. Rowley. Quadratic functions and GF(q)-groups, Proc. Amer. Math. Soc. 125 (1997), 22272237.

[24] S. E. PAYNE. An essay on skew translation generalized quadrangles, Geom. Dedicata 32 (1989), 93-118.

[25] S. E. PAYNE. Lecture at "Finite Geometries, Groups and Computation (Pingree Park, 2004)."

[26] S. E. PaYne And J. A. Thas. Finite Generalized Quadrangles, Research Notes in Mathematics 110, Pitman Advanced Publishing Program, Boston/London/Melbourne, 1984.

[27] S. E. PAYne And J. A. Thas. Finite Generalized Quadrangles. Second edition, EMS Series of Lectures in Mathematics, European Mathematical Society, 2009.

[28] S. E. PAyne And K. Thas. Notes on elation generalized quadrangles, European J. Combin. 24 (2003), 969-981.

[29] T. Penttila and C. E. Praeger. Ovoids and translation ovals J. London Math. Soc. 56 (1997), 607-624.

[30] R. Rostermundt. Elation groups of the Hermitian surface $H\left(3, q^{2}\right)$ over a finite field of characteristic 2, Innov. Incidence Geom. 5 (2007), 117-128.

[31] A. P. Sprague. Translation nets, Mitt. Math. Sem. Giessen 157 (1982), 46-68.

[32] G. STROTh. Quadratic forms and special 2-groups, Arch. Math. 33 (1979/80), 415-422.

[33] J. A. ThAs. Ovoidal translation planes, Arch. Math. 23 (1972), 110-112.

[34] J. A. Thas. Generalized quadrangles and flocks of cones, European J. Combin. 8 (1987), 441-452.

[35] J. A. Thas. Generalized quadrangles of order $\left(s, s^{2}\right)$, III, J. Combin. Theory Ser. A 87 (1999), 247-272.

[36] J. A. Thas, K. Thas And H. VAn Maldeghem. Translation Generalized Quadrangles, Series in Pure Mathematics 26, World Scientific, Singapore, 2006. 
[37] K. Thas. On symmetries and translation generalized quadrangles, Finite Geometries, Developments in Mathematics 3, Proceedings of the Fourth Isle of Thorns Conference 'Finite Geometries', 16-21 July 2000, Edited by A. Blokhuis et al., Kluwer Academic Publishers (2001), 333-345.

[38] K. THAS. A theorem concerning nets arising from generalized quadrangles with a regular point, Des. Codes Cryptogr. 25 (2002), 247-253.

[39] K. THAS. The classification of generalized quadrangles with two translation points, Beiträge Algebra Geom. 43 (2002), 365-398.

[40] K. ThAS. On translation generalized quadrangles for which the translation dual arises from a flock, Glasgow Math. J. 45 (2003), 457-474.

[41] K. Thas. Symmetry in Finite Generalized Quadrangles, Frontiers in Mathematics 1, Birkhäuser, 2004.

[42] K. THAS. Some basic questions and open problems in the theory of elation generalized quadrangles, and their solutions, Bulletin Belgian Math. Soc. — Simon Stevin 12 (2006), 909-918.

[43] K. Thas. A question of Kantor on translation quadrangles, Adv. Geom. 7 (2007), 375-378.

[44] K. Thas. Solution of a question of Knarr, Proc. Amer. Math. Soc. 136 (2008), 1409-1418.

[45] K. Thas. Order in building theory, in: Surveys in Combinatorics 2011, pp. 23-331, London Math. Soc. Lecture Note Ser. 392, Cambridge Univ. Press, Cambridge, 2011.

[46] K. Thas. Generalized quadrangles from a local point of view, J. Geom. 101 (2011), 223-238.

[47] K. Thas. A Course on Elation Quadrangles, EMS Series of Lectures in Mathematics, European Math. Soc., 2012.

[48] K. THAS. Isomorphisms of groups related to flocks, J. Algebraic Combin. 36 (2012), 111-121.

[49] K. THAS. Local half Moufang quadrangles, Des. Codes Cryptogr. 68 (2013), 319-324.

[50] K. Thas. Classification of skew translation generalized quadrangles, I, Discrete Math. Theor. Comput. Sci. 17 (2015), 89-96.

[51] K. Thas And S. E. Payne. Foundations of elation generalized quadrangles, European J. Combin. 27 (2006), $51-61$.

[52] J. TITS. Sur la trialité et certains groupes qui s'en dduisent, Inst. Hautes Etudes Sci. Publ. Math. 2 (1959), 13-60.

[53] J. TITS. Buildings of Spherical Type and Finite BN-Pairs, Lecture Notes in Mathematics 386, Springer-Verlag, Berlin-New York, 1974.

[54] J. Tits And R. M. Weiss. Moufang Polygons, Springer Monographs in Mathematics, Springer-Verlag, Berlin, 2002.

[55] H. Van Maldeghem. Generalized Polygons, Monographs in Mathematics 93, Birkhäuser-Verlag, Basel, 1998.

Ghent University, Department of Mathematics, Krijgslaan 281, S25, B-9000 Ghent, BelgiUm

E-mail address: koen. thas@gmail.com 